\title{
Three Dimensional Fractional Fourier-Mellin Transform, and its Applications
}

\author{
Arvind Kumar Sinha*, Srikumar Panda \\ Department of Mathematics, National Institute of Technology Raipur (C.G.)-492010, India
}

Received February 25, 2021; Revised May 13, 2021; Accepted June 6, 2021

Cite This Paper in the following Citation Styles

(a): [1] Arvind Kumar Sinha, Srikumar Panda, "Three Dimensional Fractional Fourier-Mellin Transform, and its Applications," Mathematics and Statistics, Vol.9, No.4, pp. 465-480, 2021. DOI: 10.13189/ms.2021.090407

(b): Arvind Kumar Sinha, Srikumar Panda, (2021). Three Dimensional Fractional Fourier-Mellin Transform, and its Applications. Mathematics and Statistics, 9(4), 465-480. DOI: 10.13189/ms.2021.090407

Copyright $@ 2021$ by authors, all rights reserved. Authors agree that this article remains permanently open access under the terms of the Creative Commons Attribution License 4.0 International License
Abstract The main objective of the paper is to study the three-dimensional fractional Fourier Mellin transforms (3DFRFMT), their basic properties and applicability due to mainly use in the radar system, reconstruction of grayscale images, in the detection of the human face, etc. Only the fractional Fourier transform is based on time-frequency distribution, whereas only the fractional Mellin transform is on scale covariant transformation. Both transforms can discover action in the definite assortment. The fractional Fourier transform is applicable for controlling the range of shift, whereas the fractional Mellin transform is accustomed to managing the range of rotation and scaling of the function. So, combining both transformations, we get an elegant expression for 3DFRFMT, which can be used in several fields. The paper introduces the concept of three-dimensional fractional Fourier Mellin transforms and their applications. Modulation property is the most useful concept in the signal system, radar tech- nology, pattern reorganization, and many more in the integral transform. Parseval's identity applies to the conservation of energy in the universe. Thus we establish the modulation the- orem, Parseval's theorem, scaling theorem, analytic theorem for three-dimensional fractional Fourier Mellin transform. We also give some examples of three-dimensional fractional Fourier-Mellin transform on some functions. Finally, we provide three-dimensional fractional Fourier-Mellin transform applications for solving homogeneous and non-homogeneous Mboctara partial differential equations that we can apply with advantages to solve the different types of problems in signal processing systems. The transform is beneficial in a maritime strategy as a co-realtor to control moments in any specific three-dimensional space. The concept is the most powerful tool to deal with any information system problems. After obtaining the generalization, we can explore many more
ideas in applying three-dimensional fractional Fourier-Mellin transformations in many real word problems.
Keywords Fractional Fourier Transform, Fractional Mellin Transform, Three Dimensional Fractional Fourier- Mellin Transform, Test Function.

\section{Introduction}
Many Mathematicians have been used fractional Integral transform to solve problems in different fields of Sciences and Technologies [1-3]. Fractional Fourier transform has been used in signal processing [4]. Fractional calculus properties and applications were proposed by applying fractional Mellin integral transform in operator theory [5]. Fourier method in initial boundary value problems for regions with curvilinear boundaries were discussed in [6] were as orthogonal splines in approximation of functions, were presented in [7] which is useful for creation of approximations of functions in form of Fourier transform.
Two dimensional fractional Mellin transform was introduced by [8] apply on some elementary functions. Two dimensional fractional Fourier transform was introduced by [9] to obtain some generalized results on some elementary functions. Two dimensional fractional Fourier-Mellin transform was introduced by [10] and established some related theorems.
Many Mathematicians gave the applications of fractional Fourier-Mellin transform in many fields [8-11]. So we intro- duce the concept of 3DFRFMT with its application to show its simplicity, effectiveness, and high accuracy. 


\section{Main Results}

Now we give the concept of three dimensional fractional Fourier-Mellin transform (3DFRFMT).

Definition 2.1. 3DFRFMT with parameters $\Lambda$ and $\hbar$ of $h(z, \ell, f, w, p, u)$ denoted by 3DFRFMT $h(z, \ell, f, w, p, u)$ performs a linear operation, given by the integral transform

$3 D F R F M T\{h(z, \ell, f, w, p, u)\}$

$=F_{\Lambda, \hbar}(\delta, \eta, \kappa, \xi, \varrho, \varphi)$

$=\int_{-\infty}^{\infty} \int_{-\infty}^{\infty} \int_{-\infty}^{\infty} \int_{0}^{\infty} \int_{0}^{\infty} \int_{0}^{\infty} h(z, \ell, f, w, p, u)$

$k_{\Lambda, \hbar}(z, \ell, f, w, p, u, \delta, \eta, \kappa, \xi, \varrho, \varphi) d z d \ell d f d w d p d u$

where,

$k_{\Lambda, \hbar}(z, \ell, f, w, p, u, \delta, \eta, \kappa, \xi, \varrho, \varphi)$

$=\sqrt{\frac{1-i \cot \Lambda}{2 \pi}} e^{\frac{i}{2 \sin \Lambda}\left[\left(z^{2}+\ell^{2}+f^{2}+\delta^{2}+\eta^{2}+\kappa^{2}\right) \cos \Lambda\right]}$

$e^{\frac{i}{2 \sin \Lambda}[-2(z \delta+\ell \eta+f \kappa)]} w \frac{2 \pi i \xi}{\sin \hbar}-1 \frac{2 \pi i \varrho}{p}-1$

$u \frac{2 \pi i \varphi}{\sin \hbar}-1 \frac{\pi i}{e \tan \hbar}\left[\xi^{2}+\varrho^{2}+\varphi^{2}+\log ^{2} w+\log ^{2} p+\log ^{2} u\right]$

$=C_{1 \Lambda} e^{i C_{2 \Lambda}\left[\left(z^{2}+\ell^{2}+f^{2}+\delta^{2}+\eta^{2}+\kappa^{2}\right) \cos \Lambda\right]}$

$e^{i C_{2 \Lambda}[-2(z \delta+\ell \eta+f \kappa)]} w^{c_{1 \hbar} i \xi-1} p^{c_{1 \hbar} i \varrho-1}$

$u^{c_{1 \hbar} i \varphi-1} e^{c_{1 \hbar} i\left[\xi^{2}+\varrho^{2}+\varphi^{2}+\log ^{2} w+\log ^{2} p+\log ^{2} u\right]}$

where, $C_{1 \Lambda}=\sqrt{\frac{1-i \cot \Lambda}{2 \pi}}, C_{2 \Lambda}=\frac{1}{2 \sin \Lambda}, C_{1 \hbar}=\frac{2 \pi}{\sin \hbar}$,

$C_{2 \hbar}=\frac{\pi}{\tan \hbar}$

for, $0<\Lambda<\frac{\pi}{2}, 0<\hbar<\frac{\pi}{2}$.

Now we explore the concept of test function in 3DFRFMT.

\section{Definition 2.2. (Text function in 3DFRFMT)}

An infinitely differentaible complex valued smooth function $\phi(z, \ell, f, w, p, u)$ on $R^{n}$ belong to $E\left(R^{n}\right)$, if for each compact set $I<S_{\varsigma, \tau}, J<S_{\mu, \varpi}$ and $K<S_{\omega, \sigma}$,

where

$$
\begin{gathered}
S_{\varsigma, \tau}=\left\{z, \ell,: z, \ell \in R^{n},|z| \leq \varsigma,|\ell| \leq \tau, \varsigma>0, \tau>0\right\}, \\
S_{\mu, \varpi}=\left\{f, w,: f, w, \in R^{n},|f| \leq \mu,|w| \leq \varpi,\right. \\
\mu>0, \varpi>0\},
\end{gathered}
$$

$$
S_{\omega, \sigma}=\left\{p, u: p, u \in R^{n},|p| \leq \omega,|u| \leq \sigma, \omega>0, \sigma>0\right\},
$$

$$
\begin{aligned}
& \Omega_{E, A, L, B, S, T}[\Upsilon(z, \ell, f, w, p, u)] \\
& =\sup _{\substack{z, \ell \in I \\
f, w \in J \\
p, u \in K}}\left|D_{z, \ell, f, w, p, u}^{E, A, L, B, S, T} \Upsilon(z, \ell, f, w, p, u)\right|<\infty .
\end{aligned}
$$

Here $E\left(R^{n}\right)$ will be called the space of all $\Upsilon(z, \ell, f, w, p, u)$ belong to $E\left(R^{n}\right)$ with compact support contained in $S_{\varsigma, \tau} \bigcap S_{\mu, \varpi} \bigcap S_{\omega, \sigma}$.

Definition 2.3. 3DFRFMT of $h(z, \ell, f, w, p, u)$ belong to $E^{\star}\left(R^{n}\right)$ can be defined by

$$
\begin{aligned}
& 3 \operatorname{DFRMT}\{h(z, \ell, f, w, p, u)\} \\
& =F_{\Lambda, \hbar}(\delta, \eta, \kappa, \xi, \varrho, \varphi) \\
& =<h(z, \ell, f, w, p, u) \\
& k_{\Lambda, \hbar}(z, \ell, f, w, p, u, \delta, \eta, \kappa, \xi, \varrho, \varphi)>.
\end{aligned}
$$

where,

$$
\begin{aligned}
& k_{\Lambda, \hbar}(z, \ell, f, w, p, u, \delta, \eta, \kappa, \xi, \varrho, \varphi) \\
& =\sqrt{\frac{1-i \cot \Lambda}{2 \pi}} e^{\frac{1}{2 \sin \Lambda}\left[\left(z^{2}+\ell^{2}+f^{2}+\delta^{2}+\eta^{2}+\kappa^{2}\right) \cos \Lambda\right]} \\
& e^{\frac{1}{2 \sin \Lambda}[-2(z \delta+\ell \eta+f \kappa)]} w \frac{2 \pi i \xi}{\sin \hbar}-1 \frac{2 \pi i \varrho}{p}-1 \\
& u \frac{2 \pi i \varphi}{\sin \hbar}-1 \frac{\pi i}{e \tan \hbar}\left[\xi^{2}+\varrho^{2}+\varphi^{2}+\log ^{2} w+\log ^{2} p+\log ^{2} u\right] \\
& =C_{1 \Lambda} e^{i C_{2 \Lambda}\left[\left(z^{2}+\ell^{2}+f^{2}+\delta^{2}+\eta^{2}+\kappa^{2}\right) \cos \Lambda-2(z \delta+\ell \eta+f \kappa)\right]} \\
& w^{c_{1 \hbar} i \xi-1} p^{c_{1 \hbar} i \varrho-1} u^{c_{1 \hbar} i \varphi-1} \\
& e^{c_{1 \hbar} i\left[\xi^{2}+\varrho^{2}+\varphi^{2}+\log ^{2} w+\log ^{2} p+\log ^{2} u\right]} \\
& =C_{1 \Lambda} e^{i C_{2 \Lambda}\left[\left(z^{2}+\ell^{2}+f^{2}+\delta^{2}+\eta^{2}+\kappa^{2}\right) \cos \Lambda\right]} \\
& e^{i C_{2 \Lambda}[-2(z \delta+\ell \eta+f \kappa)]} w^{c_{1 \hbar} i \xi-1} p^{c_{1 \hbar} i \varrho-1} u^{c_{1 \hbar} i \varphi-1} \\
& e^{c_{1 \hbar} i\left[\xi^{2}+\varrho^{2}+\varphi^{2}+\log ^{2} w+\log ^{2} p+\log ^{2} u\right]}
\end{aligned}
$$

where, $C_{1 \Lambda}=\sqrt{\frac{1-i \cot \Lambda}{2 \pi}}, C_{2 \Lambda}=\frac{1}{2 \sin \Lambda}, C_{1 \hbar}=\frac{2 \pi}{\sin \hbar}$,

$C_{2 \hbar}=\frac{\pi}{\tan \hbar}$

for, $0<\Lambda<\frac{\pi}{2}, \quad 0<\hbar<\frac{\pi}{2}$.

The above equation (2) shows the application of $h(z, \ell, f, w, p, u) \quad \in \quad E^{\star}\left(R^{n}\right)$ to 
$k_{\Lambda, \hbar}(z, \ell, f, w, p, u, \delta, \eta, \kappa, \xi, \varrho, \varphi) \in E$.

Now we give the modulation theorem in 3DFRFMT.

\section{Theorem 2.4.(a) Show that}

$$
\begin{aligned}
& 3 D F R F M T\{h(z, \ell, f, t, m, n) \\
& \cos (\alpha z+\beta \ell+\gamma f+\xi t+\nu m+\lambda n)\} \quad(\psi, \omega, \chi, \sigma, \iota, \varepsilon) \\
& =\frac{1}{2} e^{i[(z \psi+\ell \omega+f \chi) \cos \Lambda]} e^{i\left[-\frac{\alpha^{2}+\beta^{2}+\gamma^{2}}{2} \cos \Lambda \sin \Lambda\right]} \\
& 3 D F R F M T\left\{h(z, \ell, f, t, m, n) e^{i(\xi t+\nu m+\lambda n)}\right. \\
& [(\psi-\alpha \sin \Lambda),(\omega-\beta \sin \Lambda),(\chi-\gamma \sin \Lambda), \sigma, \iota, \varepsilon]\} \\
& +\frac{1}{2} e^{-i[(z \psi+\ell \omega+f \chi) \cos \Lambda]} e^{-i\left[\frac{\alpha^{2}+\beta^{2}+\gamma^{2}}{2} \cos \Lambda \sin \Lambda\right]} \\
& 3 D F R F M T\left\{h(z, \ell, f, t, m, n) e^{-i(\xi t+\nu m+\lambda n)}\right. \\
& [(\psi+\alpha \sin \Lambda),(\omega+\beta \sin \Lambda),(\chi+\gamma \sin \Lambda), \sigma, \iota, \varepsilon]\} . \\
& 3 D F R F M T\{h(z, \ell, f, t, m, n) \\
& \cos (\alpha z+\beta \ell+\gamma f+\xi t+\nu m+\lambda n)\}(\psi, \omega, \chi, \sigma, \iota, \varepsilon) \\
& =\int_{-\infty}^{\infty} \int_{-\infty}^{\infty} \int_{-\infty}^{\infty} \int_{0}^{\infty} \int_{0}^{\infty} \int_{0}^{\infty} h(z, \ell, f, t, m, n) \\
& k_{\Lambda, \hbar}(z, \ell, f, t, m, n, \psi, \omega, \chi, \sigma, \iota, \varepsilon) \\
& \cos (\alpha z+\beta \ell+\gamma f+\xi t+\nu m+\lambda n) \\
& d z d \ell d f d t d m d n \\
& =\int_{-\infty}^{\infty} \int_{-\infty}^{\infty} \int_{-\infty}^{\infty} \int_{0}^{\infty} \int_{0}^{\infty} \int_{0}^{\infty} h(z, \ell, f, t, m, n) \\
& \sqrt{\frac{1-i \cot \Lambda}{2 \pi}} e^{\frac{i}{2 \sin \Lambda}\left[\left(z^{2}+\ell^{2}+f^{2}+\psi^{2}+\omega^{2}+\chi^{2}\right) \cos \Lambda\right]} \\
& e^{\frac{i}{2 \sin \Lambda}[-2(z \psi+\ell \omega+f \chi)]} \frac{2 \pi i \sigma}{t \sin \hbar}-1 \frac{2 \pi i \iota}{m} \frac{2 \pi i \varepsilon}{\sin \hbar}-1 \frac{2 \pi}{\sin \hbar}-1 \\
& e \frac{\pi i}{\tan \hbar}\left[\sigma^{2}+\iota^{2}+\varepsilon^{2}+\log ^{2} t+\log ^{2} m+\log ^{2} n\right] \\
& \cos (\alpha z+\beta \ell+\gamma f+\xi t+\nu m+\lambda n) d z d \ell d f d t d m d n \\
& =\frac{1}{2} \int_{-\infty}^{\infty} \int_{-\infty}^{\infty} \int_{-\infty}^{\infty} \int_{0}^{\infty} \int_{0}^{\infty} \int_{0}^{\infty} h(z, \ell, f, t, m, n) \\
& \sqrt{\frac{1-i \cot \Lambda}{2 \pi}} e^{\frac{i}{2 \sin \Lambda}\left[\left(z^{2}+\ell^{2}+f^{2}+\psi^{2}+\omega^{2}+\chi^{2}\right) \cos \Lambda\right.} \\
& e^{\frac{i}{2 \sin \Lambda}[-2(z \psi+\ell \omega+f \chi)]} \\
& \frac{2 \pi i \sigma}{t \sin \hbar}-1 \frac{2 \pi i \iota}{m} \frac{2 \pi i \varepsilon}{\sin \hbar}-1 \frac{2}{\sin \hbar}-1
\end{aligned}
$$

$e^{\frac{\pi i}{\tan \hbar}\left[\sigma^{2}+\iota^{2}+\varepsilon^{2}+\log ^{2} t+\log ^{2} m+\log ^{2} n\right]}$

$e^{[i(\alpha z+\beta \ell+\gamma f+\xi t+\nu m+\lambda n)+}$

$e^{-i(\alpha z+\beta \ell+\gamma f+\xi t+\nu m+\lambda n)]}$

$d z d \ell d f d t d m d n$

$$
\begin{aligned}
& =\frac{1}{2} \sqrt{\frac{1-i \cot \Lambda}{2 \pi}} \\
& \int_{-\infty}^{\infty} \int_{-\infty}^{\infty} \int_{-\infty}^{\infty} \int_{0}^{\infty} \int_{0}^{\infty} \int_{0}^{\infty} h(z, \ell, f, t, m, n) \\
& e^{\frac{i}{2 \sin \Lambda}\left[\left(z^{2}+\ell^{2}+f^{2}+\psi^{2}+\omega^{2}+\chi^{2}\right) \cos \Lambda\right]} \\
& e^{\frac{i}{2 \sin \Lambda}[-2(z \psi+\ell \omega+f \chi)]} \\
& \frac{2 \pi i \sigma}{t \sin \hbar}-1 \frac{2 \pi i \iota}{m} \frac{\sin }{\sin }^{-1} \frac{2 \pi i \varepsilon}{n \sin \hbar}-1
\end{aligned}
$$

$e^{\frac{\pi i}{\tan \hbar}\left[\sigma^{2}+\iota^{2}+\varepsilon^{2}+\log ^{2} t+\log ^{2} m+\log ^{2} n\right]}$

$e^{\frac{\pi i}{\tan \hbar}[i(\xi t+\nu m+\lambda n)} e^{i(\alpha z+\beta \ell+\gamma f)}$

$d z d \ell d f d t d m d n+$

$$
\begin{aligned}
& \frac{1}{2} \sqrt{\frac{1-i \cot \Lambda}{2 \pi}} \\
& \int_{-\infty}^{\infty} \int_{-\infty}^{\infty} \int_{-\infty}^{\infty} \int_{0}^{\infty} \int_{0}^{\infty} \int_{0}^{\infty} h(z, \ell, f, t, m, n) \\
& e^{\frac{i}{2 \sin \Lambda}\left[\left(z^{2}+\ell^{2}+f^{2}+\psi^{2}+\omega^{2}+\chi^{2}\right) \cos \Lambda\right]} \\
& e \frac{i}{2 \sin \Lambda}[-2(z \psi+\ell \omega+f \chi)] \\
& \frac{2 \pi i \sigma}{t \sin \hbar}-1 \frac{2 \pi i \iota}{m} \frac{\sin \hbar}{n}_{n} \frac{2 \pi i \varepsilon}{\sin \hbar}-1 \\
& e^{\frac{\pi i}{\tan \hbar}\left[\sigma^{2}+\iota^{2}+\varepsilon^{2}+\log ^{2} t+\log ^{2} m+\log ^{2} n\right]}
\end{aligned}
$$

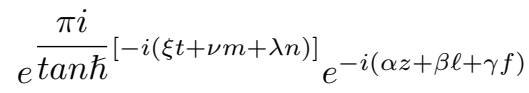

$$
\begin{aligned}
& d z d \ell d f d t d m d n \\
& =\frac{1}{2} \sqrt{\frac{1-i \cot \Lambda}{2 \pi}} e^{\frac{i}{2}\left(\psi^{2}+\omega^{2}+\chi^{2}\right) \cot \Lambda} \\
& \int_{-\infty}^{\infty} \int_{-\infty}^{\infty} \int_{-\infty}^{\infty} \int_{0}^{\infty} \int_{0}^{\infty} \int_{0}^{\infty} h(z, \ell, f, t, m, n)
\end{aligned}
$$




$$
\begin{aligned}
& e^{\frac{i}{2} z^{2} \cot \Lambda-i(\Psi \operatorname{cosec} \Lambda-\alpha) z} \\
& e^{\frac{i}{\ell^{2} \cot \Lambda-i(\omega \operatorname{cosec} \Lambda-\beta) \ell}} \\
& e^{\frac{i}{2} f^{2} \cot \Lambda-i(\chi \operatorname{cosec} \Lambda-\gamma) f} \\
& \frac{2 \pi i \sigma}{t \sin \hbar}-1 \frac{2 \pi i \iota}{m} \frac{\sin \hbar}{n}_{n} \frac{2 \pi i \varepsilon}{\sin \hbar}-1 \\
& e^{\frac{\pi i}{\tan \hbar}\left[\sigma^{2}+\iota^{2}+\varepsilon^{2}+\log ^{2} t+\log ^{2} m+\log ^{2} n\right]} \\
& e^{\frac{\pi i}{\tan \hbar}[i(\xi t+\nu m+\lambda n)]} d z d \ell d f d t d m d n \\
& +\frac{1}{2} \sqrt{\frac{1-i \cot \Lambda}{2 \pi}} e^{\frac{i}{2}\left(\psi^{2}+\omega^{2}+\chi^{2}\right) \cot \Lambda} \\
& \int_{-\infty}^{\infty} \int_{-\infty}^{\infty} \int_{-\infty}^{\infty} \int_{0}^{\infty} \int_{0}^{\infty} \int_{0}^{\infty} h(z, \ell, f, t, m, n) \\
& e^{\frac{i}{z^{2} \cot \Lambda-i(\Psi \operatorname{cosec} \Lambda+\alpha) z}} \\
& e^{\frac{i}{2} \ell^{2} \cot \Lambda-i(\omega \operatorname{cosec} \Lambda+\beta) \ell} \\
& e^{\frac{i}{2} f^{2} \cot \Lambda-i(\chi \operatorname{cosec} \Lambda+\gamma) f} \\
& \frac{2 \pi i \sigma}{t \sin \hbar}-1 \frac{2 \pi i \iota}{m}-1 \frac{2 \pi i \varepsilon}{\sin \hbar}-1 \\
& e^{\frac{\pi i}{\tan \hbar}\left[\sigma^{2}+\iota^{2}+\varepsilon^{2}+\log ^{2} t+\log ^{2} m+\log ^{2} n\right]} \\
& e^{\frac{\pi i}{\tan \hbar}[-i(\xi t+\nu m+\lambda n)} d z d \ell d f d t d m d n \\
& =\frac{1}{2} \sqrt{\frac{1-i \cot \Lambda}{2 \pi}} e^{\frac{i}{2}\left(\psi^{2}+\omega^{2}+\chi^{2}\right) \cot \Lambda} \\
& \int_{-\infty}^{\infty} \int_{-\infty}^{\infty} \int_{-\infty}^{\infty} \int_{0}^{\infty} \int_{0}^{\infty} \int_{0}^{\infty} h(z, \ell, f, t, m, n) \\
& e^{\frac{i}{2} z^{2} \cot \Lambda-i \operatorname{cosec} \Lambda(\Psi-\alpha \sin \Lambda) z} \\
& e^{\frac{i}{2} \ell^{2} \cot \Lambda-i \operatorname{cosec} \Lambda(\omega-\beta \sin \Lambda) \ell} \\
& e^{\frac{i}{2} f^{2} \cot \Lambda-i \operatorname{cosec} \Lambda(\chi-\gamma \sin \Lambda) f} \\
& \frac{2 \pi i \sigma}{t \sin \hbar}-1 \frac{2 \pi i \iota}{m} \frac{\sin }{\sin }-1_{n} \frac{2 \pi i \varepsilon}{\sin \hbar}-1 \\
& e^{\frac{\pi i}{\tan \hbar}\left[\sigma^{2}+\iota^{2}+\varepsilon^{2}+\log ^{2} t+\log ^{2} m+\log ^{2} n\right]}
\end{aligned}
$$$$
e^{\frac{\pi i}{\tan \hbar}[i(\xi t+\nu m+\lambda n)]} \quad d z d \ell d f d t d m d n
$$$$
+\frac{1}{2} \sqrt{\frac{1-i \cot \Lambda}{2 \pi}} e^{\frac{i}{2}\left(\psi^{2}+\omega^{2}+\chi^{2}\right) \cot \Lambda}
$$$$
\int_{-\infty}^{\infty} \int_{-\infty}^{\infty} \int_{-\infty}^{\infty} \int_{0}^{\infty} \int_{0}^{\infty} \int_{0}^{\infty} h(z, \ell, f, t, m, n)
$$$$
e^{\frac{i}{2} z^{2} \cot \Lambda-i \operatorname{cosec} \Lambda(\Psi+\alpha \sin \Lambda) z}
$$$$
e^{\frac{i}{\ell^{2} \cot \Lambda-i \operatorname{cosec} \Lambda(\omega+\beta \sin \Lambda) \ell}}
$$$$
e^{\frac{i}{2} f^{2} \cot \Lambda-i \operatorname{cosec} \Lambda(\chi+\gamma \sin \Lambda) f}
$$$$
\frac{2 \pi i \sigma}{\sin \hbar}-1 \frac{2 \pi i \iota}{m} \frac{\sin \hbar}{n}_{n} \frac{2 \pi i \varepsilon}{\sin \hbar}-1
$$$$
\frac{\pi i}{\tan \hbar}\left[\sigma^{2}+\iota^{2}+\varepsilon^{2}+\log ^{2} t+\log ^{2} m+\log ^{2} n\right]
$$$$
e^{\frac{\pi i}{\tan \hbar}[-i(\xi t+\nu m+\lambda n)]} d z d \ell d f d t d m d n
$$$$
=\frac{1}{2} \sqrt{\frac{1-i \cot \Lambda}{2 \pi}}
$$$$
e^{i\left[(\alpha \Psi+\beta \omega+\gamma \chi) \cos \Lambda-\frac{\alpha^{2}+\beta^{2}+\gamma^{2}}{2} \cos \Lambda \sin \Lambda\right]}
$$$$
\int_{-\infty}^{\infty} \int_{-\infty}^{\infty} \int_{-\infty}^{\infty} \int_{0}^{\infty} \int_{0}^{\infty} \int_{0}^{\infty} h(z, \ell, f, t, m, n)
$$$$
e^{\frac{i}{[}\left[z^{2}+(\Psi-\alpha \sin \Lambda)^{2}\right] \cot \Lambda} e^{-i \operatorname{cosec} \Lambda(\Psi-\alpha \sin \Lambda) z}
$$$$
e^{\frac{i}{2}\left[\ell^{2}+(\omega-\beta \sin \Lambda)^{2}\right] \cot \Lambda} e^{-i \operatorname{cosec} \Lambda(\omega-\beta \sin \Lambda) \ell}
$$$$
e^{\frac{i}{2}\left[f^{2}+(\chi-\gamma \sin \Lambda)^{2}\right] \cot \Lambda} e^{-i \operatorname{cosec} \Lambda(\chi-\gamma \sin \Lambda) f}
$$$$
\frac{2 \pi i \sigma}{\sin \hbar}-1 \frac{2 \pi i \iota}{m} \frac{2 \pi i n}{\sin }^{-1} \frac{2 \pi i \varepsilon}{\sin \hbar}-1
$$$$
e^{\frac{\pi i}{\tan \hbar}\left[\sigma^{2}+\iota^{2}+\varepsilon^{2}+\log ^{2} t+\log ^{2} m+\log ^{2} n\right]}
$$$$
e^{\frac{\pi i}{\tan \hbar}[i(\xi t+\nu m+\lambda n)]} d z d \ell d f d t d m d n
$$$$
+\frac{1}{2} \sqrt{\frac{1-i \cot \Lambda}{2 \pi}} e^{-i[(\alpha \Psi+\beta \omega+\chi \gamma) \cos \Lambda]}
$$$$
e^{-i\left[\frac{\alpha^{2}+\beta^{2}+\gamma^{2}}{2} \cos \Lambda \sin \Lambda\right]}
$$ 


$$
\begin{aligned}
& \int_{-\infty}^{\infty} \int_{-\infty}^{\infty} \int_{-\infty}^{\infty} \int_{0}^{\infty} \int_{0}^{\infty} \int_{0}^{\infty} h(z, \ell, f, t, m, n) \\
& e^{\frac{i}{2}\left[z^{2}+(\Psi+\alpha \sin \Lambda)^{2}\right] \cot \Lambda} e^{-i \operatorname{cosec} \Lambda(\Psi+\alpha \sin \Lambda) z} \\
& e^{\frac{i}{2}\left[\ell^{2}+(\omega+\beta \sin \Lambda)^{2}\right] \cot \Lambda} e^{-i \operatorname{cosec} \Lambda(\omega+\beta \sin \Lambda) \ell} \\
& e^{\frac{i}{2}\left[f^{2}+(\chi+\gamma \sin \Lambda)^{2}\right] \cot \Lambda} e^{-i \operatorname{cosec} \Lambda(\chi+\gamma \sin \Lambda) f} \\
& \frac{2 \pi i \sigma}{\sin \hbar}-1 \frac{2 \pi i \iota}{m} \frac{\sin }{\sin }^{-1} \frac{2 \pi i \varepsilon}{\sin \hbar}-1 \\
& e^{\frac{\pi i}{\tan \hbar}\left[\sigma^{2}+\iota^{2}+\varepsilon^{2}+\log ^{2} t+\log ^{2} m+\log ^{2} n\right]}
\end{aligned}
$$

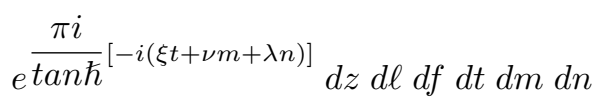

$$
\begin{aligned}
& =\frac{1}{2} \sqrt{\frac{1-i \cot \Lambda}{2 \pi}} e^{i[(\alpha \Psi+\beta \omega+\chi) \cos \Lambda]} \\
& e^{i\left[-\frac{\alpha^{2}+\beta^{2}+\gamma^{2}}{2} \cos \Lambda \sin \Lambda\right]} \\
& \int_{-\infty}^{\infty} \int_{-\infty}^{\infty} \int_{-\infty}^{\infty} \int_{0}^{\infty} \int_{0}^{\infty} \int_{0}^{\infty} h(z, \ell, f, t, m, n) \\
& e^{\frac{i}{2}\left[z^{2}+\ell^{2}+f^{2}\right] \cot \Lambda} \\
& e^{\frac{i}{2}\left\{\left[(\Psi-\alpha \sin \Lambda)^{2}+(\omega-\beta \sin \Lambda)^{2}+(\chi-\gamma \sin \Lambda)^{2}\right] \cot \Lambda\right\}} \\
& \frac{2 \pi i \sigma}{t \sin \hbar}-1 \frac{2 \pi i \iota}{m} \frac{2 \pi i \varepsilon}{\sin \hbar}-1 \frac{2}{\sin \hbar}-1 \\
& e \frac{\pi i}{\tan \hbar}\left[\sigma^{2}+\iota^{2}+\varepsilon^{2}+\log ^{2} t+\log ^{2} m+\log ^{2} n\right] \\
& e^{+i(\xi t+\nu m+\lambda n)} d z d \ell d f d t d m d n
\end{aligned}
$$$$
+\frac{1}{2} \sqrt{\frac{1-i \cot \Lambda}{2 \pi}} e^{-i[(\alpha \Psi+\beta \omega+\chi \gamma) \cos \Lambda]}
$$$$
e^{-i\left[\frac{\alpha^{2}+\beta^{2}+\gamma^{2}}{2} \cos \Lambda \sin \Lambda\right]}
$$$$
\int_{-\infty}^{\infty} \int_{-\infty}^{\infty} \int_{-\infty}^{\infty} \int_{0}^{\infty} \int_{0}^{\infty} \int_{0}^{\infty} h(z, \ell, f, t, m, n)
$$$$
e^{\frac{i}{2}\left[z^{2}+\ell^{2}+f^{2}\right] \cot \Lambda}
$$$$
e^{\frac{i}{2}\left\{\left[(\Psi+\alpha \sin \Lambda)^{2}+(\omega+\beta \sin \Lambda)^{2}+(\chi+\gamma \sin \Lambda)^{2}\right] \cot \Lambda\right\}}
$$$$
e^{\frac{i}{2}\{-2 \operatorname{cosec} \Lambda[(\Psi+\alpha \sin \Lambda) z+(\omega+\beta \sin \Lambda) \ell+(\chi+\gamma \sin \Lambda) f]\}}
$$

$$
\begin{aligned}
& t \frac{2 \pi i \sigma}{\sin \hbar}-1 \frac{2 \pi i \iota}{m \sin \hbar}-1 \frac{2 \pi i \varepsilon}{n \sin \hbar}-1 \\
& e^{\frac{\pi i}{\tan \gamma}\left[\sigma^{2}+\iota^{2}+\varepsilon^{2}+\log ^{2} t+\log ^{2} m+\log ^{2} n\right]} \\
& e^{-i(\xi t+\nu m+\lambda n)} d z d \ell d f d t d m d n \\
& =\frac{1}{2} e^{i\left[(z \psi+\ell \omega+f \chi) \cos \Lambda-\frac{\alpha^{2}+\beta^{2}+\gamma^{2}}{2} \cos \Lambda \sin \Lambda\right]} \\
& 3 D F R F M T\left\{h(z, \ell, f, t, m, n) e^{i(\xi t+\nu m+\lambda n)}\right. \\
& [(\psi-\alpha \sin \Lambda),(\omega-\beta \sin \Lambda),(\chi-\gamma \sin \Lambda), \sigma, \iota, \varepsilon]\} \\
& +\frac{1}{2} e^{-i\left[(z \psi+\ell \omega+f \chi) \cos \Lambda+\frac{\alpha^{2}+\beta^{2}+\gamma^{2}}{2} \cos \Lambda \sin \Lambda\right]} \\
& 3 D F R F M T\left\{h(z, \ell, f, t, m, n) e^{-i(\xi t+\nu m+\lambda n)}\right. \\
& [(\psi+\alpha \sin \Lambda),(\omega+\beta \sin \Lambda),(\chi+\gamma \sin \Lambda), \sigma, \iota, \varepsilon]\} .
\end{aligned}
$$

Theorem 2.4.(b) Show that

$$
\begin{aligned}
& 3 D F R F M T\{h(z, \ell, f, t, m, n) \sin (\alpha z+ \\
& \beta \ell+\gamma f+\xi t+\nu m+\lambda n)\}(\psi, \omega, \chi, \sigma, \iota, \varepsilon) \\
& =\frac{1}{2 i} e^{i[(z \psi+\ell \omega+f \chi) \cos \Lambda} \\
& e^{i\left[-\frac{\alpha^{2}+\beta^{2}+\gamma^{2}}{2} \cos \Lambda \sin \Lambda\right]} \\
& 3 D F R F M T\left\{h(z, \ell, f, t, m, n) e^{i(\xi t+\nu m+\lambda n)}\right. \\
& [(\psi-\alpha \sin \Lambda),(\omega-\beta \sin \Lambda),(\chi-\gamma \sin \Lambda), \sigma, \iota, \varepsilon]\} \\
& \quad-\frac{1}{2 i} e^{-i[(z \psi+\ell \omega+f \chi) \cos \Lambda]} \\
& e^{-i\left[\frac{\alpha^{2}+\beta^{2}+\gamma^{2}}{2} \cos \Lambda \sin \Lambda\right]}
\end{aligned}
$$

$3 D F R F M T\left\{h(z, \ell, f, t, m, n) e^{-i(\xi t+\nu m+\lambda n)}\right.$

$[(\psi+\alpha \sin \Lambda),(\omega+\beta \sin \Lambda),(\chi+\gamma \sin \Lambda), \sigma, \iota, \varepsilon]\}$

\section{Proof: We have}

$$
\begin{aligned}
& 3 \text { DFR RT }\{h(z, \ell, f, t, m, n) \sin (\alpha z+ \\
& \beta e+\gamma f+\xi t+\nu m+\lambda n)\}(\psi, \omega, \chi, \sigma, \iota, \varepsilon) \\
& =\int_{-\infty}^{\infty} \int_{-\infty}^{\infty} \int_{-\infty}^{\infty} \int_{0}^{\infty} \int_{0}^{\infty} \int_{0}^{\infty} h(z, \ell, f, t, m, n) \\
& \quad k_{\Lambda, \hbar}(z, \ell, f, t, m, n, \psi, \omega, \chi, \sigma, \iota, \varepsilon) \\
& \quad \sin (\alpha z+\beta \ell+\gamma f+\xi t+\nu m+\lambda n) \\
& \quad d z d \ell d f d t d m d n
\end{aligned}
$$




$$
=\int_{-\infty}^{\infty} \int_{-\infty}^{\infty} \int_{-\infty}^{\infty} \int_{0}^{\infty} \int_{0}^{\infty} \int_{0}^{\infty} h(z, \ell, f, t, m, n)
$$$$
\sqrt{\frac{1-i \cot \Lambda}{2 \pi}} e^{\frac{i}{2 \sin \Lambda}\left[\left(z^{2}+\ell^{2}+f^{2}+\psi^{2}+\omega^{2}+\chi^{2}\right) \cos \Lambda\right]}
$$$$
e^{\frac{i}{2 \sin \Lambda}[-2(z \psi+\ell \omega+f \chi)]}
$$$$
\frac{2 \pi i \sigma}{\sin \hbar}-1 \frac{2 \pi i \iota}{m} \frac{\sin }{\sin }^{-1} \frac{2 \pi i \varepsilon}{\sin \hbar}-1
$$$$
e^{\frac{\pi i}{\tan \hbar}\left[\sigma^{2}+\iota^{2}+\varepsilon^{2}+\log ^{2} t+\log ^{2} m+\log ^{2} n\right]}
$$$$
\sin (\alpha z+\beta \ell+\gamma f+\xi t+\nu m+\lambda n)
$$

$d z d \ell d f d t d m d n$

$$
=\frac{1}{2 i} \sqrt{\frac{1-i \cot \Lambda}{2 \pi}}
$$

$\int_{-\infty}^{\infty} \int_{-\infty}^{\infty} \int_{-\infty}^{\infty} \int_{0}^{\infty} \int_{0}^{\infty} \int_{0}^{\infty} h(z, \ell, f, t, m, n)$

$e^{\frac{i}{2 \sin \Lambda}\left[\left(z^{2}+\ell^{2}+f^{2}+\psi^{2}+\omega^{2}+\chi^{2}\right) \cos \Lambda\right.}$

$$
\begin{aligned}
& e^{\frac{i}{2 \sin \Lambda}[-2(z \psi+e \omega+f \chi)]} \\
& \frac{2 \pi i \sigma}{\sin \hbar}-1 \frac{2 \pi i \iota}{m}-1 \frac{2 \pi i \varepsilon}{\sin \hbar}-1 \\
& \sin \hbar
\end{aligned}
$$$$
\frac{\pi i}{\tan \hbar}\left[\sigma^{2}+\iota^{2}+\varepsilon^{2}+\log ^{2} t+\log ^{2} m+\log ^{2} n\right]
$$$$
e^{\frac{\pi i}{\tan \hbar}[i(\xi t+\nu m+\lambda n)]} e^{i(\alpha z+\beta \ell+\gamma f)}
$$

$d z d \ell d f d t d m d n$

$$
\begin{aligned}
& -\frac{1}{2 i} \sqrt{\frac{1-i \cot \Lambda}{2 \pi}} \\
& \int_{-\infty}^{\infty} \int_{-\infty}^{\infty} \int_{-\infty}^{\infty} \int_{0}^{\infty} \int_{0}^{\infty} \int_{0}^{\infty} h(z, \ell, f, t, m, n) \\
& e^{\frac{i}{2 \sin \Lambda}\left[\left(z^{2}+\ell^{2}+f^{2}+\psi^{2}+\omega^{2}+\chi^{2}\right) \cos \Lambda\right]} \\
& e \frac{i}{2 \sin \Lambda}[-2(z \psi+\ell \omega+f \chi)] \\
& \frac{2 \pi i \sigma}{\operatorname{sin\hbar }}-1 \frac{2 \pi i \iota}{m \operatorname{sin\hbar }}-1 \frac{2 \pi i \varepsilon}{\sin ^{2}-1} \\
& e \frac{\pi i}{\tan \hbar}\left[\sigma^{2}+\iota^{2}+\varepsilon^{2}+\log ^{2} t+\log ^{2} m+\log ^{2} n\right] \\
& e^{\frac{\pi i}{\tan \hbar}[-i(\xi t+\nu m+\lambda n)]} e^{-i(\alpha z+\beta \ell+\gamma f)}
\end{aligned}
$$

$d z d \ell d f d t d m d n$

$$
\begin{aligned}
& =\frac{1}{2 i} \sqrt{\frac{1-i \cot \Lambda}{2 \pi}} e^{\frac{i}{2}\left(\psi^{2}+\omega^{2}+\chi^{2}\right) \cot \Lambda} \\
& \int_{-\infty}^{\infty} \int_{-\infty}^{\infty} \int_{-\infty}^{\infty} \int_{0}^{\infty} \int_{0}^{\infty} \int_{0}^{\infty} h(z, \ell, f, t, m, n) \\
& e^{\frac{i}{2} z^{2} \cot \Lambda-i(\Psi \operatorname{cosec} \Lambda-\alpha) z} \\
& e^{\frac{i}{2} \ell^{2} \cot \Lambda-i(\omega \operatorname{cosec} \Lambda-\beta) \ell} \\
& e^{\frac{i}{2} f^{2} \cot \Lambda-i(\chi \operatorname{cosec} \Lambda-\gamma) f}
\end{aligned}
$$$$
\frac{2 \pi i \sigma}{\sin \hbar}-1 \frac{2 \pi i \iota}{m} \frac{\sin \hbar}{n}^{-1} \frac{2 \pi i \varepsilon}{\sin \hbar}-1
$$$$
e^{\frac{\pi i}{\tan \hbar}\left[\sigma^{2}+\iota^{2}+\varepsilon^{2}+\log ^{2} t+\log ^{2} m+\log ^{2} n\right]}
$$

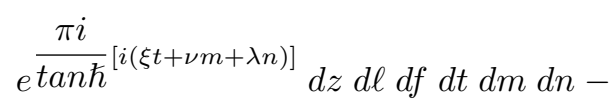

$$
\begin{aligned}
& \frac{1}{2 i} \sqrt{\frac{1-i \cot \Lambda}{2 \pi}} e^{\frac{i}{2}\left(\psi^{2}+\omega^{2}+\chi^{2}\right) \cot \Lambda} \\
& \int_{-\infty}^{\infty} \int_{-\infty}^{\infty} \int_{-\infty}^{\infty} \int_{0}^{\infty} \int_{0}^{\infty} \int_{0}^{\infty} h(z, \ell, f, t, m, n) \\
& e \frac{i}{2} z^{2} \cot \Lambda-i(\Psi \operatorname{cosec} \Lambda+\alpha) z \\
& e \frac{i}{2} \ell^{2} \cot \Lambda-i(\omega \operatorname{cosec} \Lambda+\beta) \ell \\
& e^{\frac{i}{2} f^{2} \cot \Lambda-i(\chi \operatorname{cosec} \Lambda+\gamma) f}
\end{aligned}
$$

$$
\frac{2 \pi i \sigma}{\sin \hbar}-1 \frac{2 \pi i \iota}{m}-1 \frac{2 \pi i \varepsilon}{\sin \hbar}-1
$$$$
e^{\frac{\pi i}{\tan \hbar}\left[\sigma^{2}+\iota^{2}+\varepsilon^{2}+\log ^{2} t+\log ^{2} m+\log ^{2} n\right]}
$$

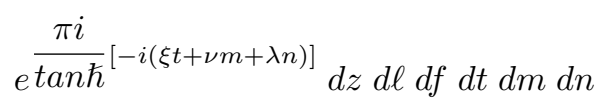$$
=\frac{1}{2 i} \sqrt{\frac{1-i \cot \Lambda}{2 \pi}}
$$$$
\int_{-\infty}^{\infty} \int_{-\infty}^{\infty} \int_{-\infty}^{\infty} \int_{0}^{\infty} \int_{0}^{\infty} \int_{0}^{\infty} h(z, \ell, f, t, m, n)
$$

$e^{\frac{i}{2}\left[(\Psi-\alpha \sin \Lambda)^{2}\right] \cot \Lambda} e^{i \Psi \alpha \cos \Lambda}$

$e^{\frac{-i \alpha^{2}}{2} \cos \Lambda \sin \Lambda} e^{\frac{i}{2} z^{2} \cot \Lambda-i \operatorname{cosec} \Lambda(\Psi-\alpha \sin \Lambda) z}$ 
$e^{\frac{i}{2\left[(\omega-\beta \sin \Lambda)^{2}\right] \cot \Lambda}} e^{i \beta \omega \cos \Lambda}$

$e^{\frac{-i \beta^{2}}{2} \cos \Lambda \sin \Lambda} e^{\frac{i}{2^{2} \cot \Lambda-i \operatorname{cosec} \Lambda(\omega-\beta \sin \Lambda) \ell}}$ $e^{\frac{i}{2\left[(\chi-\gamma \sin \Lambda)^{2}\right] \cot \Lambda}} e^{i \chi \gamma \cos \Lambda} e^{\frac{-i \gamma^{2}}{2} \cos \Lambda \sin \Lambda}$

$e^{\frac{1}{2} f^{2} \cot \Lambda-i \operatorname{cosec} \Lambda(\chi-\gamma \sin \Lambda) f}$

$\frac{2 \pi i \sigma}{t \sin \hbar}-1 \frac{2 \pi i \iota}{m} \frac{\sin \hbar}{n}_{n} \frac{2 \pi i \varepsilon}{\sin \hbar}-1$

$\left.e^{\frac{\pi i}{\tan \hbar}\left[\sigma^{2}+\iota^{2}+\varepsilon^{2}+\log ^{2} t+\log ^{2} m+\log ^{2}\right.} n\right]$

$e^{\frac{\pi i}{\tan \hbar}[i(\xi t+\nu m+\lambda n)} d z d \ell d f d t d m d n$

$-\frac{1}{2 i} \sqrt{\frac{1-i \cot \Lambda}{2 \pi}}$

$\int_{-\infty}^{\infty} \int_{-\infty}^{\infty} \int_{-\infty}^{\infty} \int_{0}^{\infty} \int_{0}^{\infty} \int_{0}^{\infty} h(z, \ell, f, t, m, n)$

$e^{\frac{i}{2^{\left[(\Psi+\alpha \sin \Lambda)^{2}\right] \cot \Lambda}} e^{-i \Psi \alpha \cos \Lambda}}$

$e^{\frac{-i \alpha^{2}}{2} \cos \Lambda \sin \Lambda} e^{\frac{1}{2} z^{2} \cot \Lambda-i \operatorname{cosec} \Lambda(\Psi+\alpha \sin \Lambda) z}$

$e^{\frac{i}{2\left[(\omega+\beta \sin \Lambda)^{2}\right] \cot \Lambda}} e^{-i \beta \omega \cos \Lambda} e^{\frac{-i \beta^{2}}{2} \cos \Lambda \sin \Lambda}$

$e^{\frac{i}{2} \ell^{2} \cot \Lambda-i \operatorname{cosec} \Lambda(\omega+\sin \Lambda) \ell}$

$e^{\frac{i}{2}\left[(\chi+\gamma \sin \Lambda)^{2}\right] \cot \Lambda} e^{-i \chi \gamma \cos \Lambda} e^{\frac{-i \gamma^{2}}{2} \cos \Lambda \sin \Lambda}$

$e^{\frac{i}{2} f^{2} \cot \Lambda-i \operatorname{cosec} \Lambda(\chi+\gamma \sin \Lambda) f}$

$\frac{2 \pi i \sigma}{t \sin \hbar}-1 \frac{2 \pi i \iota}{m} \frac{\sin }{\sin }^{-1} \frac{2 \pi i \varepsilon}{\sin \hbar}-1$

$e^{\frac{\pi i}{\tan \hbar}\left[\sigma^{2}+\iota^{2}+\varepsilon^{2}+\log ^{2} t+\log ^{2} m+\log ^{2} n\right]}$

$e^{\frac{\pi i}{\tan \hbar}[-i(\xi t+\nu m+\lambda n)]} d z d \ell d f d t d m d n$

$=\frac{1}{2 i} \sqrt{\frac{1-i \cot \Lambda}{2 \pi}}$ $e^{i\left[(\alpha \Psi+\beta \omega+\chi \gamma) \cos \Lambda-\frac{\alpha^{2}+\beta^{2}+\gamma^{2}}{2} \cos \Lambda \sin \Lambda\right]}$

$\int_{-\infty}^{\infty} \int_{-\infty}^{\infty} \int_{-\infty}^{\infty} \int_{0}^{\infty} \int_{0}^{\infty} \int_{0}^{\infty} h(z, \ell, f, t, m, n)$

$e^{\frac{i}{2}\left\{\left[z^{2}+\ell^{2}+f^{2}+(\Psi-\alpha \sin \Lambda)^{2}+(\omega-\beta \sin \Lambda)^{2}+(\chi-\gamma \sin \Lambda)^{2}\right\}\right.}$

$e^{\frac{i}{2}\{-2 \operatorname{cosec} \Lambda[(\Psi-\alpha \sin \Lambda) z+(\omega-\beta \sin \Lambda) \ell+(\chi-\gamma \sin \Lambda) f]\}}$

$\frac{2 \pi i \sigma}{\sin \hbar}-1 \frac{2 \pi i \iota}{m} \frac{2 \pi i \varepsilon}{\sin \hbar}^{n} \frac{2 \pi i n}{\sin \hbar}-1$

$e^{\frac{\pi i}{\tan \hbar}\left[\sigma^{2}+\iota^{2}+\varepsilon^{2}+\log ^{2} t+\log ^{2} m+\log ^{2} n\right]}$

$e^{+i(\xi t+\nu m+\lambda n)} d z d \ell d f d t d m d n$

$-\frac{1}{2 i} \sqrt{\frac{1-i \cot \Lambda}{2 \pi}}$

$e^{-i\left[(\alpha \Psi+\beta \omega+\chi \gamma) \cos \Lambda+\frac{\alpha^{2}+\beta^{2}+\gamma^{2}}{2} \cos \Lambda \sin \Lambda\right]}$

$e^{\frac{i}{2}\left\{\left[z^{2}+\ell^{2}+f^{2}+(\Psi+\alpha \sin \Lambda)^{2}+(\omega+\beta \sin \Lambda)^{2}+(\chi+\gamma \sin \Lambda)^{2}\right\}\right.}$
$e^{\frac{i}{2}\{-2 \operatorname{cosec} \Lambda[(\Psi+\alpha \sin \Lambda) z+(\omega+\beta \sin \Lambda) \ell+(\chi+\gamma \sin \Lambda) f]\}}$

$\frac{2 \pi i \sigma}{t \sin \hbar}-1 \frac{2 \pi i \iota}{m}-1 \frac{2 \pi i \varepsilon}{\sin \hbar}-1$

$e^{\frac{\pi i}{\tan \hbar}\left[\sigma^{2}+\iota^{2}+\varepsilon^{2}+\log ^{2} t+\log ^{2} m+\log ^{2} n\right]}$

$e^{-i(\xi t+\nu m+\lambda n)]} d z d \ell d f d t d m d n$

$=\frac{1}{2 i} e^{i[(\alpha \psi+\beta \omega+\gamma \chi) \cos \Lambda]}$

$e^{i\left[-\frac{\alpha^{2}+\beta^{2}+\gamma^{2}}{2} \cos \Lambda \sin \Lambda\right]}$

$3 D F R F M T\left\{h(z, \ell, f, t, m, n) e^{i(\xi t+\nu m+\lambda n)}\right.$

$[(\psi-\alpha \sin \Lambda),(\omega-\beta \sin \Lambda),(\chi-\gamma \sin \Lambda), \sigma, \iota, \varepsilon]\}$

$-\frac{1}{2 i} e^{-i[(z \psi+\ell \omega+f \chi) \cos \Lambda}$

$e^{-i\left[\frac{\alpha^{2}+\beta^{2}+\gamma^{2}}{2} \cos \Lambda \sin \Lambda\right]}$

$3 D F R F M T\left\{h(z, \ell, f, t, m, n) e^{-i(\xi t+\nu m+\lambda n)}\right.$

$[(\psi+\alpha \sin \Lambda),(\omega+\beta \sin \Lambda),(\chi+\gamma \sin \Lambda), \sigma, \iota, \varepsilon]\}$

Now we give Parseval's theorem in 3DFRFMT. 


\section{Theorem 2.5. If}

$$
\begin{aligned}
& 3 D F R F M T\{h(z, \ell, f, t, m, n)\} \\
& =F_{\Lambda, \hbar}(\psi, \omega, \chi, \sigma, \iota, \varepsilon) \\
& \quad \text { and } \\
& 3 D F R F M T\{g(z, \ell, f, t, m, n)\} \\
& =G_{\Lambda, \hbar}(\psi, \omega, \chi, \sigma, \iota, \varepsilon)
\end{aligned}
$$

Show that

$$
\text { (i) } \int_{-\infty}^{\infty} \int_{-\infty}^{\infty} \int_{-\infty}^{\infty} \int_{0}^{\infty} \int_{0}^{\infty} \int_{0}^{\infty} h(z, \ell, f, t, m, n)
$$

$\overline{g(z, \ell, f, t, m, n)} d z d \ell d f d t d m d n$

$$
=\int_{-\infty}^{\infty} \int_{-\infty}^{\infty} \int_{-\infty}^{\infty} \int_{0}^{\infty} \int_{0}^{\infty} \int_{0}^{\infty} F_{\Lambda, \hbar}(\psi, \omega, \chi, \sigma, \iota, \varepsilon)
$$

$\overline{G_{\Lambda, \hbar}(\psi, \omega, \chi, \sigma, \iota, \varepsilon)} d \psi d \omega d \chi d \sigma d \iota d \varepsilon$

(ii) $\int_{-\infty}^{\infty} \int_{-\infty}^{\infty} \int_{-\infty}^{\infty} \int_{0}^{\infty} \int_{0}^{\infty} \int_{0}^{\infty}|h(z, \ell, f, t, m, n)|^{2}$ $d z d \ell d f d t d m d n$

$=\int_{-\infty}^{\infty} \int_{-\infty}^{\infty} \int_{-\infty}^{\infty} \int_{0}^{\infty} \int_{0}^{\infty} \int_{0}^{\infty}\left|F_{\Lambda, \hbar}(\psi, \omega, \chi, \sigma, \iota, \varepsilon)\right|^{2}$

$d \psi d \omega d \chi d \sigma d \iota d \varepsilon$

proof. We have

$$
3 D F R F M T\{g(z, \ell, f, t, m, n)\}
$$$$
(\psi, \omega, \chi, \sigma, \iota, \varepsilon)
$$$$
=G_{\Lambda, \hbar}(\psi, \omega, \chi, \sigma, \iota, \varepsilon)
$$$$
=\int_{-\infty}^{\infty} \int_{-\infty}^{\infty} \int_{-\infty}^{\infty} \int_{0}^{\infty} \int_{0}^{\infty} \int_{0}^{\infty} g(z, \ell, f, t, m, n)
$$$$
\sqrt{\frac{1-i \cot \Lambda}{2 \pi}} e^{\frac{i}{2 \sin \Lambda}\left[\left(z^{2}+\ell^{2}+f^{2}+\psi^{2}+\omega^{2}+\chi^{2}\right)\right] \cos \Lambda}
$$$$
e^{\frac{i}{2 \sin \Lambda}[-2(z \psi+\ell \omega+f \chi)]}
$$$$
\frac{2 \pi i \sigma}{t \operatorname{sin\hbar }}-1 \frac{2 \pi i \iota}{m} \frac{\sin }{\sin }^{-1} \frac{2 \pi i \varepsilon}{n \sin \hbar}-1
$$$$
e^{\frac{\pi i}{\tan \hbar}\left[\sigma^{2}+\iota^{2}+\varepsilon^{2}+\log ^{2} t+\log ^{2} m+\log ^{2} n\right]} d z d \ell d f d t d m d n
$$

By using inversion formula for three dimensional fractional Fourier-Mellin transform $g(z, \ell, f, t, m, n)$

$$
=\frac{1}{8 \pi^{3}} \int_{-\infty}^{\infty} \int_{-\infty}^{\infty} \int_{-\infty}^{\infty} \int_{0}^{\infty} \int_{0}^{\infty} \int_{0}^{\infty} \frac{1}{\sin ^{3} \Lambda \sin ^{3} \hbar}
$$

$$
\begin{aligned}
& G_{\Lambda, \hbar}(\psi, \omega, \chi, \sigma, \iota, \varepsilon)\left[\frac{1-i \cot \Lambda}{2 \pi}\right]^{-\frac{1}{2}} \\
& e^{-\frac{i}{2 \sin \Lambda}\left[\left(z^{2}+\ell^{2}+f^{2}+\psi^{2}+\omega^{2}+\chi^{2}\right) \cos \Lambda-2(z \psi+\ell \omega+f \chi)\right]} \\
& \quad t^{-\frac{2 \pi i \sigma}{\sin \hbar} m}-\frac{2 \pi i \iota}{\sin \hbar} n-\frac{2 \pi i \varepsilon}{\sin \hbar} \\
& e^{-\frac{\pi i}{\tan \hbar}\left[\sigma^{2}+\iota^{2}+\varepsilon^{2}+\log ^{2} t+\log ^{2} m+\log ^{2} n\right]}
\end{aligned}
$$

$d \psi d \omega d \chi d \sigma d \iota d \varepsilon$

$$
\begin{gathered}
=\frac{1}{8 \pi^{3}} \int_{-\infty}^{\infty} \int_{-\infty}^{\infty} \int_{-\infty}^{\infty} \int_{0}^{\infty} \int_{0}^{\infty} \int_{0}^{\infty} \frac{1}{\sin ^{3} \Lambda \sin ^{3} \hbar} \\
G_{\Lambda, \hbar}(\psi, \omega, \chi, \sigma, \iota, \varepsilon)\left[\frac{1-i \cot \Lambda}{2 \pi}\right]^{-\frac{1}{2}} \\
e^{-\frac{i}{2}\left[\left(z^{2}+\ell^{2}+f^{2}\right)\right] \cot \Lambda} e^{-\frac{i}{2}\left[\left(\psi^{2}+\omega^{2}+\chi^{2}\right)\right] \cot \Lambda}
\end{gathered}
$$

$e^{i[(z \psi+\ell \omega+f \chi)] \operatorname{cosec} \Lambda} t^{-\frac{2 \pi i \sigma}{\sin \hbar}} m-\frac{2 \pi i \iota}{\sin \hbar} n^{-\frac{2 \pi i \varepsilon}{\sin \gamma}}$

$e^{-\frac{\pi i}{\tan \hbar}\left[\sigma^{2}+\iota^{2}+\varepsilon^{2}\right]} e^{-\frac{\pi i}{\tan \hbar}\left[\log ^{2} t+\log ^{2} m+\log ^{2} n\right]}$

$d \psi d \omega d \chi d \sigma d \iota d \varepsilon$

$$
=\frac{1}{(2 \pi) \frac{5}{2} \sin ^{3} \Lambda \sin ^{3} \hbar \sqrt{1-i \cot \Lambda}}
$$

$e^{-\frac{i}{2}\left[\left(z^{2}+\ell^{2}+f^{2}\right)\right] \cot \Lambda} e^{-\frac{\pi i}{\tan \hbar}\left[\log ^{2} t+\log ^{2} m+\log ^{2} n\right]}$

$\int_{-\infty}^{\infty} \int_{-\infty}^{\infty} \int_{-\infty}^{\infty} \int_{0}^{\infty} \int_{0}^{\infty} \int_{0}^{\infty} G_{\Lambda, \hbar}(\psi, \omega, \chi, \sigma, \iota, \varepsilon)$

$e^{-\frac{i}{2}\left[\left(\psi^{2}+\omega^{2}+\chi^{2}\right)\right] \cot \Lambda}$

$e^{i[(z \psi+\ell \omega+f \chi)] \operatorname{cosec} \Lambda} t^{-\frac{2 \pi i \sigma}{\sin \hbar}} m-\frac{2 \pi i \iota}{\sin \hbar}$

$n^{-\frac{2 \pi i \varepsilon}{\sin \hbar}} e^{-\frac{\pi i}{\tan \hbar}\left[\sigma^{2}+\iota^{2}+\varepsilon^{2}\right]}$

$d \psi d \omega d \chi d \sigma d \iota d \varepsilon$

Taking complex conjugate of above term

$$
\begin{aligned}
& \overline{g(z, \ell, f, t, m, n)} \\
& =\frac{5}{\frac{5}{2}}
\end{aligned}
$$


$e^{\frac{i}{2}\left[\left(z^{2}+\ell^{2}+f^{2}\right)\right] \cot \Lambda}$

$e^{\frac{\pi i}{\tan \hbar}\left[\log ^{2} t+\log ^{2} m+\log ^{2} n\right]}$

$\int_{-\infty}^{\infty} \int_{-\infty}^{\infty} \int_{-\infty}^{\infty} \int_{0}^{\infty} \int_{0}^{\infty} \int_{0}^{\infty} \overline{G_{\Lambda, \hbar}(\psi, \omega, \chi, \sigma, \iota, \varepsilon)}$

$e^{\frac{i}{2}\left[\left(\psi^{2}+\omega^{2}+\chi^{2}\right)\right] \cot \Lambda} e^{-i[(z \psi+\ell \omega+f \chi)] \operatorname{cosec} \Lambda}$

$\frac{2 \pi i \sigma}{\sin \hbar} \frac{2 \pi i \iota}{m} \frac{2 \pi i \varepsilon}{\sin \hbar} \frac{\pi i}{\sin \hbar}\left[\sigma^{2}+\iota^{2}+\varepsilon^{2}\right]$

$d \psi d \omega d \chi d \sigma d \iota d \varepsilon$

Now consider,

$$
\begin{aligned}
& \int_{-\infty}^{\infty} \int_{-\infty}^{\infty} \int_{-\infty}^{\infty} \int_{0}^{\infty} \int_{0}^{\infty} \int_{0}^{\infty} h(z, \ell, f, t, m, n) \\
& \overline{g(z, \ell, f, t, m, n)} d z d \ell d f d t d m d n \\
& =\int_{-\infty}^{\infty} \int_{-\infty}^{\infty} \int_{-\infty}^{\infty} \int_{0}^{\infty} \int_{0}^{\infty} \int_{0}^{\infty} h(z, \ell, f, t, m, n) \\
& d z d \ell d f d t d m d n \\
& \left\{\frac{1}{5}\right. \\
& (2 \pi) \overline{2} \sin ^{3} \Lambda \sin ^{3} \hbar \sqrt{1+\cot \Lambda} \\
& e^{\frac{i}{2}\left[\left(z^{2}+\ell^{2}+f^{2}\right)\right] \cot \Lambda} \frac{\pi i}{e \operatorname{tan\hbar }}\left[\log ^{2} t+\log ^{2} m+\log ^{2} n\right] \\
& \int_{-\infty}^{\infty} \int_{-\infty}^{\infty} \int_{-\infty}^{\infty} \int_{0}^{\infty} \int_{0}^{\infty} \int_{0}^{\infty} \overline{g_{\Lambda, \hbar}(\psi, \omega, \chi, \sigma, \iota, \varepsilon)} \\
& e^{\frac{i}{2\left[\left(\Psi^{2}+\omega^{2}+\chi^{2}\right)\right] \cot \Lambda}} e^{-i[(z \psi+\ell \omega+f \chi)] \operatorname{cosec} \Lambda} \\
& \frac{2 \pi i \sigma}{t \sin \hbar} \quad \frac{2 \pi i \iota}{\sin \hbar} n \frac{2 \pi i \varepsilon}{\sin \hbar} \frac{\pi i}{\tan ^{2}}\left[\sigma^{2}+\iota^{2}+\varepsilon^{2}\right] \\
& d \psi d \omega d \chi d \sigma d \iota d \varepsilon\}
\end{aligned}
$$$$
\begin{array}{r}
\int_{-\infty}^{\infty} \int_{-\infty}^{\infty} \int_{-\infty}^{\infty} \int_{0}^{\infty} \int_{0}^{\infty} \int_{0}^{\infty} \frac{1}{(2 \pi) \overline{2} \sin ^{3} \Lambda \sin ^{3} \hbar} \\
\sqrt{1+\cot \Lambda}
\end{array}
$$

$\overline{G_{\Lambda, \hbar}(\psi, \omega, \chi, \sigma, \iota, \varepsilon)} d \psi d \omega d \chi d \sigma d \iota d \varepsilon$

$$
\begin{aligned}
& \int_{-\infty}^{\infty} \int_{-\infty}^{\infty} \int_{-\infty}^{\infty} \int_{0}^{\infty} \int_{0}^{\infty} \int_{0}^{\infty} h(z, \ell, f, t, m, n) \\
& e^{\frac{i}{2}\left[\left(z^{2}+\ell^{2}+f^{2}+\psi^{2}+\omega^{2}+\chi^{2}\right) \cot \Lambda\right.} \\
& \frac{i}{[-2(z \psi+\ell \omega+f \chi) \operatorname{cosec} \Lambda]}
\end{aligned}
$$

$$
\begin{aligned}
& t \frac{2 \pi i \sigma}{\sin \hbar} \quad \frac{2 \pi i \iota}{\sin \hbar} \frac{2 \pi i \varepsilon}{n \sin \hbar} \\
& e^{\frac{\pi i}{\tan \hbar}\left[\sigma^{2}+\iota^{2}+\varepsilon^{2}+\log ^{2} t+\log ^{2} m+\log ^{2} n\right]}
\end{aligned}
$$

$d z d \ell d f d t d m d n$

$$
\begin{aligned}
& =\frac{(2 \pi)^{\frac{1}{2}}}{(2 \pi)^{\frac{5}{2}} \sin ^{3} \Lambda \sin ^{3} \hbar \sqrt{1-i^{2} \cot ^{2} \Lambda}} \\
& \int_{-\infty}^{\infty} \int_{-\infty}^{\infty} \int_{-\infty}^{\infty} \int_{0}^{\infty} \int_{0}^{\infty} \int_{0}^{\infty} \overline{G_{\Lambda, \hbar}(\psi, \omega, \chi, \sigma, \iota, \varepsilon)}
\end{aligned}
$$

$d \psi d \omega d \chi d \sigma d \iota d \varepsilon$

$$
\begin{aligned}
& \int_{-\infty}^{\infty} \int_{-\infty}^{\infty} \int_{-\infty}^{\infty} \int_{0}^{\infty} \int_{0}^{\infty} \int_{0}^{\infty} \sqrt{\frac{1-i \cot \Lambda}{2 \pi}} h(z, \ell, f, t, m, n) \\
& e^{\frac{i}{2 \sin \Lambda}\left[\left(z^{2}+\ell^{2}+f^{2}+\psi^{2}+\omega^{2}+\chi^{2}\right) \cos \Lambda\right.} \\
& e^{\frac{i}{2 \sin \Lambda}[-2(z \psi+\ell \omega+f \chi)]}
\end{aligned}
$$$$
(t, m, n) t^{\frac{2 \pi i \sigma}{\sin \hbar}}-1 \frac{2 \pi i \iota}{\sin \hbar}^{-1} \frac{2 \pi i \varepsilon}{\sin \hbar}^{-1}
$$$$
e^{\frac{\pi i}{\tan \hbar}\left[\sigma^{2}+\iota^{2}+\varepsilon^{2}+\log ^{2} t+\log ^{2} m+\log ^{2} n\right]}
$$

$d z d \ell d f d t d m d n$

$$
=\frac{(2 \pi)^{\frac{1}{2}}}{(2 \pi)^{\frac{5}{2}} \sin ^{3} \Lambda \sin ^{3} \hbar \sqrt{1-i^{2} \cot ^{2} \Lambda}}
$$

$\int_{-\infty}^{\infty} \int_{-\infty}^{\infty} \int_{-\infty}^{\infty} \int_{0}^{\infty} \int_{0}^{\infty} \int_{0}^{\infty} \overline{g(z, \ell, f, t, m, n)}$

$[(t m n) h(z, \ell, f, t, m, n)]$

$(\psi, \omega, \chi, \sigma, \iota, \varepsilon) d \psi d \omega d \chi d \sigma d \iota d \varepsilon$

$$
=\frac{1}{(2 \pi)^{2} \sin ^{3} \Lambda \sin ^{3} \hbar \sqrt{1+\cot ^{2} \Lambda}}
$$

$\int_{-\infty}^{\infty} \int_{-\infty}^{\infty} \int_{-\infty}^{\infty} \int_{0}^{\infty} \int_{0}^{\infty} \int_{0}^{\infty} \overline{g_{\Lambda, \hbar}(\psi, \omega, \chi, \sigma, \iota, \varepsilon)}$

$F_{\Lambda, \hbar}[(t m n) h(z, \ell, f, t, m, n)](\psi, \omega, \chi, \sigma, \iota, \varepsilon)$

$d \psi d \omega d \chi d \sigma d \iota d \varepsilon$

$$
\begin{aligned}
& =\frac{1}{(2 \pi)^{2} \sin ^{3} \Lambda \sin ^{3} \hbar \operatorname{cosec} \Lambda} \\
& \int_{-\infty}^{\infty} \int_{-\infty}^{\infty} \int_{-\infty}^{\infty} \int_{0}^{\infty} \int_{0}^{\infty} \int_{0}^{\infty} \overline{g_{\Lambda, \hbar}(\psi, \omega, \chi, \sigma, \iota, \varepsilon)}
\end{aligned}
$$




$$
\begin{aligned}
& F_{\Lambda, \hbar}[(t m n) h(z, \ell, f, t, m, n)] \\
& =\frac{1}{(2 \pi)^{2} \sin ^{2} \Lambda \sin ^{3} \hbar} \\
& F_{\Lambda, \hbar}[(t m n) h(z, \ell, f, t, m, n)] \\
& (\psi, \omega, \chi, \sigma, \iota, \varepsilon) \\
& d \psi d \omega d \chi d \sigma d \iota d \varepsilon \\
& \text { Putting } g(z, \ell, f, t, m, n) \\
& =h(z, \ell, f, t, m, n) \text {, } \\
& F_{\Lambda, \hbar}(\psi, \omega, \chi, \sigma, \iota, \varepsilon) \\
& =G_{\Lambda, \hbar}(\psi, \omega, \chi, \sigma, \iota, \varepsilon), \\
& \overline{F_{\Lambda, \hbar}(\psi, \omega, \chi, \sigma, \iota, \varepsilon)} \\
& =\overline{G_{\Lambda, \hbar}(\psi, \omega, \chi, \sigma, \iota, \varepsilon)}
\end{aligned}
$$$$
(\psi, \omega, \chi, \sigma, \iota, \varepsilon) d \psi d \omega d \chi d \sigma d \iota d \varepsilon
$$$$
\int_{-\infty}^{\infty} \int_{-\infty}^{\infty} \int_{-\infty}^{\infty} \int_{0}^{\infty} \int_{0}^{\infty} \int_{0}^{\infty} \overline{g_{\Lambda, \hbar}(\psi, \omega, \chi, \sigma, \iota, \varepsilon)}
$$

By using above result

$$
\begin{aligned}
& \int_{-\infty}^{\infty} \int_{-\infty}^{\infty} \int_{-\infty}^{\infty} \int_{0}^{\infty} \int_{0}^{\infty} \int_{0}^{\infty} h(z, \ell, f, t, m, n) \\
& \overline{g(z, \ell, f, t, m, n)} \\
& =\int_{-\infty}^{\infty} \int_{-\infty}^{\infty} \int_{-\infty}^{\infty} \int_{0}^{\infty} \int_{0}^{\infty} \int_{0}^{\infty} F_{\Lambda, \hbar}(\psi, \omega, \chi, \sigma, \iota, \varepsilon) \\
& \overline{G_{\Lambda, \hbar}(\psi, \omega \chi, \sigma, \iota, \varepsilon)}
\end{aligned}
$$

Therefore,

$$
\int_{-\infty}^{\infty} \int_{-\infty}^{\infty} \int_{-\infty}^{\infty} \int_{0}^{\infty} \int_{0}^{\infty} \int_{0}^{\infty}|h(z, \ell, f, t, m, n)|^{2}
$$

$d z d \ell d f d t d m d n$

$$
=\int_{-\infty}^{\infty} \int_{-\infty}^{\infty} \int_{-\infty}^{\infty} \int_{0}^{\infty} \int_{0}^{\infty} \int_{0}^{\infty}\left|F_{\Lambda, \hbar}(\psi, \omega, \chi, \sigma, \iota, \varepsilon)\right|^{2}
$$

$d \psi d \omega d \chi d \sigma d \iota d \varepsilon$

Now we give scaling theorem in 3DFRFMT.

\section{Theorem 2.6.}

$$
3 D F R F M T\{h(\alpha z, \beta \ell, \gamma z, \xi t, \nu m, \wp n)\}
$$

$$
\begin{aligned}
& (\psi, \omega, \chi, \sigma, \iota, \varsigma) \\
= & \frac{1}{\alpha \beta \gamma} \xi^{-\frac{2 \pi i \sigma}{\sin \hbar}} \nu \frac{-2 \pi i \iota}{\sin \hbar} \wp \frac{-2 \pi i \varsigma}{\sin \hbar}
\end{aligned}
$$

$$
e^{\frac{\pi i}{\tan \hbar}\left(\log ^{2} \xi+\log ^{2} \nu+\log ^{2} \wp\right)}
$$

$\left\{F R M T e^{\frac{-2 \pi i}{\tan \hbar}(\log t \xi+\log m \nu+\log n \wp)} h(G, k, B)\right\}$

$(\sigma, \iota, \varepsilon)\{F R F T \mho(z, \ell, f)\}(\Psi, \omega, \chi)$.

where,

$\mho(z, \ell, f)$

$=e^{\frac{i}{2}\left\{\frac{1-\alpha}{\alpha}\left[\frac{1+\alpha}{\alpha}(\alpha z)^{2} \cot \hbar-2 t(\alpha z) \operatorname{cosec} \hbar\right]\right.}$

$e^{\frac{i}{2}\left\{\frac{1-\beta}{\beta}\left[\frac{1+\beta}{\beta}(\beta \ell)^{2} \cot \hbar-2 m(\beta \ell) \operatorname{cosec} \hbar\right]\right\}}$

$e^{\frac{i}{2}\left\{\frac{1-\gamma}{\gamma}\left[\frac{1+\gamma}{\gamma}(\gamma f)^{2} \cot \hbar-2 n(\gamma f) \operatorname{cosec} \hbar\right]\right\}}$

Proof: Consider

$3 D F R F M T\{h(\alpha z, \beta \ell, \gamma f, \xi t, \nu m, \lambda n)\}$

$(\psi, \omega, \chi, \sigma, \iota, \varsigma)$

$=\int_{-\infty}^{\infty} \int_{-\infty}^{\infty} \int_{-\infty}^{\infty} \int_{0}^{\infty} \int_{0}^{\infty} \int_{0}^{\infty} c_{1 \lambda}$

$e^{\frac{i}{2 \sin \lambda}\left[\left(z^{2}+\ell^{2}+f^{2}+\psi^{2}+\omega^{2}+\chi^{2}\right) \cos \lambda-2(z \psi+\ell \omega+f \chi)\right]}$

$h(\alpha z, \beta \ell, \gamma z, \xi t, \nu m, \lambda n) K_{\hbar}(t, m, n, \psi, \omega, \chi)$

$d z d \ell d f d t d m d n$

putting,

$$
\begin{aligned}
& \alpha z=A \Rightarrow \alpha d z=d A \Rightarrow d z=\frac{d A}{\alpha} \\
& \beta \ell=M \Rightarrow \beta d \ell=d M \Rightarrow d \ell=\frac{d M}{\beta} \\
& \gamma f=H \Rightarrow \gamma d f=d H \Rightarrow d f=\frac{d H}{\gamma} \\
& \xi t=G \Rightarrow \xi d t=d G \Rightarrow d t=\frac{d G}{\xi} \\
& \nu m=K \Rightarrow \nu d M=d K \Rightarrow d M=\frac{d K}{\nu} \\
& \wp n=B \Rightarrow \wp d n=d B \Rightarrow d n=\frac{d B}{\wp}
\end{aligned}
$$

Therefore,

$3 D F R F M T\{h(\alpha z, \beta \ell, \gamma f, \xi t, \nu m, \wp n)\}$

$(\psi, \omega, \chi, \sigma, \iota, \varsigma)$ 


$$
\begin{aligned}
& =c_{1 \lambda} e^{\frac{i}{2}\left(\Psi^{2}+\omega^{2}+\chi^{2}\right) \cot \lambda} \\
& \int_{-\infty}^{\infty} \int_{-\infty}^{\infty} \int_{-\infty}^{\infty} \int_{0}^{\infty} \int_{0}^{\infty} \int_{0}^{\infty} h(A, M, H, G, K, B) \\
& e^{\frac{i}{2}\left(\frac{A^{2}}{\alpha^{2}}+\frac{M^{2}}{\beta^{2}}+\frac{H^{2}}{\gamma^{2}}\right) \cot \lambda-i\left(\frac{A}{\alpha} \Psi+\frac{M}{\beta} \omega+\frac{H}{\gamma} \chi\right) \operatorname{cosec} \lambda} \\
& \left(\frac{G}{\xi}\right)^{\frac{2 \pi i \sigma}{\sin \hbar}}-1\left(\frac{K}{\nu}\right)^{\frac{2 \pi i \iota}{\sin \hbar}}-1\left(\frac{B}{\wp}\right) \frac{2 \pi i \varsigma}{\sin \hbar}-1 \\
& e^{\frac{\pi i}{\tan \hbar}\left(\sigma^{2}+\iota^{2}+\varsigma^{2}+\log ^{2} \frac{G}{\xi}+\log ^{2} \frac{K}{\nu}+\log ^{2} \frac{B}{\wp}\right)} \\
& \frac{d G}{\xi} \frac{d K}{\nu} \frac{d B}{\wp} \frac{d A}{\alpha} \frac{d M}{\beta} \frac{d H}{\gamma} \\
& =\frac{c_{1 \lambda}}{\alpha \beta \gamma \xi \nu \wp} e^{\frac{i}{2}\left(\Psi^{2}+\omega^{2}+\chi^{2}\right) \cot \lambda} \\
& \int_{-\infty}^{\infty} \int_{-\infty}^{\infty} \int_{-\infty}^{\infty} \int_{0}^{\infty} \int_{0}^{\infty} \int_{0}^{\infty} h(A, M, H, G, K, B) \\
& e^{\frac{i}{2}\left(1+\frac{1-\alpha^{2}}{\alpha^{2}}\right) \psi^{2} \cot \lambda} e^{\frac{i}{2}\left(1+\frac{1-\beta^{2}}{\alpha^{2}}\right) \omega^{2} \cot \lambda}
\end{aligned}
$$

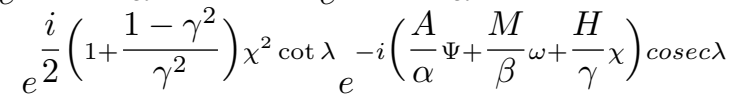

$$
\begin{aligned}
& \frac{1}{\xi \frac{2 \pi i \sigma}{\sin \hbar}-1 \frac{2 \pi i \iota}{\nu \sin \hbar}-1 \frac{2 \pi i \varsigma}{\sin \hbar}-1} \\
& e^{\frac{\pi i}{\tan \hbar}\left(\sigma^{2}+\iota^{2}+\varsigma^{2}\right)} e^{\frac{\pi i}{\tan \hbar}\left(\log ^{2} G+\log ^{2} K+\log ^{2} B\right)} \\
& e^{\frac{\pi i}{\tan \hbar}(-2 \log G \log \xi-2 \log K \log \nu-2 \log B \log \wp)} \\
& e^{\frac{\pi i}{\tan \hbar}\left(\log ^{2} \xi+\log ^{2} \nu+\log ^{2} \wp\right)} \\
& d A d M d H d G d K d B
\end{aligned}
$$$$
=\frac{c_{1 \lambda}}{\alpha \beta \gamma} e^{\frac{i}{2}\left(\Psi^{2}+\omega^{2}+\chi^{2}\right) \cot \lambda}
$$$$
\int_{-\infty}^{\infty} \int_{-\infty}^{\infty} \int_{-\infty}^{\infty} \int_{0}^{\infty} \int_{0}^{\infty} \int_{0}^{\infty} h(A, M, H, G, K, B)
$$$$
e^{\frac{i}{2}\left(\Psi^{2}+\omega^{2}+\chi^{2}\right) \cot \lambda} e^{-i(A \Psi+M \omega+H \chi) \operatorname{cosec} \lambda}
$$

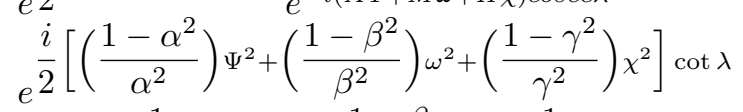$$
e^{-i\left[A \Psi\left(\frac{1-\alpha}{\alpha}\right)+M \omega\left(\frac{1-\beta}{\beta}\right)+H \chi\left(\frac{1-\gamma}{\gamma}\right)\right] \operatorname{cosec} \lambda}
$$$$
\wp-\frac{2 \pi i \varsigma}{\sin \lambda} \xi^{-\frac{2 \pi i \sigma}{\sin \lambda}} \nu-\frac{2 \pi i \iota}{\sin \lambda}
$$$$
e^{\frac{\pi i}{\tan \hbar}\left(\log ^{2} \xi+\log ^{2} \nu+\log ^{2} \lambda\right)}
$$$$
e^{\frac{\pi i}{\tan \hbar}\left(\sigma^{2}+\iota^{2}+\varsigma^{2}+\log ^{2} G+\log ^{2} K+\log ^{2} B\right)}
$$$$
e^{-\frac{2 \pi i}{\tan \hbar}(-2 \log G \log \xi-2 \log K \log \nu-2 \log B \log \lambda)}
$$$$
d A d M d H d G d K d B
$$

$$
\begin{aligned}
& =\frac{1}{\alpha \beta \gamma} \xi^{-\frac{2 \pi i \sigma}{\sin \hbar}} \nu \frac{-2 \pi i \iota}{\sin \hbar} \wp \frac{-2 \pi i \varsigma}{\sin \hbar} \\
& e^{\frac{\pi i}{\tan \hbar}\left(\log ^{2} \xi+\log ^{2} \nu+\log ^{2} \wp\right)} \\
& \left\{F R M T e^{\frac{-2 \pi i}{\tan \hbar}(\log t \xi+\log m \nu+\log n \wp)} h(G, K, B)\right\} \\
& \\
& (\sigma, \iota, \varepsilon)\{F R F T \mho(z, \ell, f)\}(\Psi, \omega, \chi) .
\end{aligned}
$$

Now we give analytic theorem on 3DFRFMT.

\section{Theorem 2.7.}

Let $h(z, \ell, f, w, p, u) \in E\left(R^{n}\right)$ and its fractional Fourier Mellin transform can be defined by

$$
\begin{aligned}
& \text { 3DFRFMT }\{h(z, \ell, f, w, p, u)\} \\
& =F_{\lambda, \hbar}(\delta, \eta, \kappa, \xi, \varrho, \varphi) \\
& =<h(z, \ell, f, w, p, u), K_{\lambda, \hbar}(\delta, \eta, \kappa, \xi, \varrho, \varphi)>.
\end{aligned}
$$

Then $F_{\lambda, \hbar}(\delta, \eta, \kappa, \xi, \varrho, \varphi)$ is analytic on $C^{n}$,

if the Supp $h \subset S_{\varsigma, \tau} \cap S_{\mu, \varpi} \cap S_{\omega, \sigma}$

where,

$$
\begin{aligned}
S_{\varsigma, \tau}= & \left\{z, \ell,: z, \ell \in R^{n},\right. \\
& |z| \leq \varsigma,|\ell| \leq \tau, \varsigma>0, \tau>0\}, \\
S_{\mu, \varpi}= & \left\{f, w,: f, w, \in R^{n},\right. \\
& |f| \leq \mu,|w| \leq \varpi,, \mu>0, \varpi>0\}, \\
S_{\omega, \sigma}= & \left\{p, u: p, u \in R^{n},\right. \\
& |p| \leq \omega,|u| \leq \sigma, \omega>0, \sigma>0\},
\end{aligned}
$$

Moreover, $F_{\lambda, \hbar}(\delta, \eta, \kappa, \xi, \varrho, \varphi)$ is differentiable and

$$
\begin{aligned}
& D_{\delta, \eta, \kappa}^{E, A, L}\left\{F_{\lambda, \hbar}(\delta, \eta, \kappa, \xi, \varrho, \varphi)\right\} \\
& =<h(z, \ell, f, w, p, u), \\
& D_{\delta, \eta, \kappa}^{E, A, L}\left\{K_{\lambda, \hbar}(z, \ell, f, w, p, u)\right\}>.
\end{aligned}
$$

\section{Proof: Let}

$$
\begin{aligned}
& \delta:\left(\delta_{1}, \delta_{2},--\delta_{p},--\delta_{E}\right) \in C^{n}, \\
& \eta:\left(\eta_{1}, \eta_{2},---\eta_{q},---\eta_{A}\right) \in C^{n}, \\
& \kappa:\left(\kappa_{1}, \eta_{2},---\eta_{r},---\eta_{L}\right) \in C^{n} .
\end{aligned}
$$


We first prove that

$$
\begin{aligned}
& \frac{\partial}{\partial \delta_{p}}\left\{F_{\lambda, \hbar}(\delta, \eta, \kappa, \xi, \varrho, \varphi)\right\} \\
& =<h(z, \ell, f, w, p, u), \\
& \frac{\partial}{\partial \delta_{p}} K_{\lambda, \hbar}(\delta, \eta, \kappa, \xi, \varrho, \varphi)>.
\end{aligned}
$$

For fixed $\delta_{p} \neq 0$, choose two concentric circle $C_{1}, C_{2}$ with center $\delta_{p}$ with radii $R_{1}$ and $R_{2}$ respectively such that $0<R_{1}<R_{2}<\left|\delta_{p}\right|$, let $\delta_{p}$ be a complex increment satisfying $0<\left|\delta_{p}\right|<R_{1}$.

\section{Consider,}

$$
\begin{aligned}
& \frac{F_{\lambda, \hbar}\left(\delta_{p}+\Delta \delta_{p}, \eta, \kappa, \xi, \varrho, \varphi\right)-F_{\lambda, \hbar}(\delta, \eta, \kappa, \xi, \varrho, \varphi)}{\delta_{p}} \\
& \quad-<h(z, \ell, f, w, p, u), \\
& \frac{\partial}{\partial \delta_{p}}\left\{K_{\lambda, \hbar}(z, \ell, f, w, p, u, \delta, \eta, \kappa, \xi, \varrho, \varphi)\right\}> \\
& =<h(z, \ell, f, w, p, u), \Psi_{\Delta \delta_{p}}(z, \ell, f, w, p, u)>
\end{aligned}
$$

where,

$$
\begin{aligned}
& \Psi_{\Delta \delta_{p}}(z, \ell, f, w, p, u) \\
& =\frac{1}{\Delta \delta_{p}}\left\{K _ { \lambda , \hbar } \left(z, \ell, f, w, p, u, \delta_{p}+\right.\right. \\
& \left.\left.\Delta \delta_{p}, \eta, \kappa, \xi, \varrho, \varphi\right)\right\}- \\
& K_{\lambda, \hbar}\left(z, \ell, f, w, p, u, \delta_{p}, \eta, \kappa, \xi, \varrho, \varphi\right) \\
& -\frac{\partial}{\partial \delta_{p}}\left\{K_{\lambda, \hbar}(z, \ell, f, w, p, u, \delta, \eta, \kappa, \xi, \varrho, \varphi)\right\}
\end{aligned}
$$

for any index $z, \ell, f \in R^{n}$ and index

integer $E=E_{1}, E_{2}-----, E_{n}$

$$
\begin{aligned}
& D_{z}^{E}\left\{K_{\lambda, \hbar}(z, \ell, f, w, p, u, \delta, \eta, \kappa, \xi, \varrho, \varphi)\right\} \\
& =D_{z}^{E}\left\{C_{1 \lambda} \beta_{1}(\ell) \beta_{2}(f)\right. \\
& \left.e^{i C_{2 \lambda}\left[\left(z^{2}+\delta^{2}\right) \cos \lambda-2 z \delta\right]} \beta_{3}(w, p, u)\right\} \\
& \text { where, } C_{1 \lambda}=\sqrt{\frac{1-i \cot \lambda}{2 \pi}}, \\
& C_{2 \lambda}=\frac{1}{2 \sin \lambda}, C_{1 \hbar}=\frac{2 \pi}{\sin \hbar} \\
& C_{2 \hbar}=\frac{\pi}{\tan \hbar} \\
& \beta_{1}(\ell)=e^{i C_{2 \lambda}\left[\left(\ell^{2}+\eta^{2}\right) \cos \lambda\right]-2 \ell \eta} \\
& \beta_{2}(f)=e^{i C_{2 \lambda}\left[\left(f^{2}+\kappa^{2}\right) \cos \lambda\right]-2 f \kappa}
\end{aligned}
$$

$$
\begin{aligned}
& \beta_{3}(w, p, u)=w^{C_{1 \hbar} i \xi-1} p^{C_{1 \hbar} i \varrho-1} \\
& u^{C_{1 \hbar} i \varphi-1} \\
& e^{C_{1 \hbar} i\left[\xi^{2}+\varrho^{2}+\varphi^{2}+\log ^{2} w+\log ^{2} p+\log ^{u}\right]} \\
& =C_{1 \alpha} \beta_{1}(\ell) \beta_{2}(f) \sum_{r=0}^{E} C_{E} \\
& C_{\alpha}(x \cos \alpha-\xi)^{E-2 r} e^{(E-2 r) u} \\
& \beta_{3}(w, p, u)
\end{aligned}
$$

where,

$$
\begin{aligned}
& C_{E}=\frac{E !}{r !(E-2 r) !}(2 i)^{E-r} \\
& C_{\alpha^{\prime}}=(\cos \alpha)^{r}\left(C_{2 \alpha}\right)^{E-r} \\
& u=i C_{2 \alpha}\left[\left(x^{2}+\xi^{2}\right) \cos \alpha-2 x \xi\right]
\end{aligned}
$$

since for any index $x \in R^{n}$,

index $\mathrm{m}$ and $o<\hbar \leqslant \frac{\pi}{2}$,

$D_{z}^{E}\left\{K_{\lambda, \hbar}(z, \ell, f, w, p, u, \delta, \eta, \kappa, \xi, \varrho, \varphi)\right\}$

is analytic inside and on $C^{\prime}$,

we have by cauchy's integral formula

$$
\begin{aligned}
& D_{z}^{E} \mho_{\Delta_{p}}(z, \ell, f, w, p, u) \\
& =\frac{1}{2 \pi i} \int_{C_{1}} D_{z}^{E}\left\{K_{\lambda, \hbar}(z, \ell, f, w, p, u\right. \\
& \left.\left.\quad \delta_{p}, \eta, \kappa, \xi, \varrho, \varphi\right)\right\} \\
& {\left[\frac{1}{\Delta \delta_{p}}\left(\frac{1}{\mho-\delta_{p}-\Delta \delta_{p}}-\frac{1}{\mho-\delta_{p}}-\frac{1}{\left(\mho-\delta_{p}\right)^{2}}\right)\right] d \mho}
\end{aligned}
$$

where, $\tilde{\delta}=\delta_{1}, \delta_{2},---\delta_{p-1}, \mho,---\delta_{n}$

$=\frac{\Delta \delta_{p}}{2 \pi i} \int_{C_{1}} \frac{A(z, \ell, f, w, p, u, \tilde{\delta}, \eta, \kappa, \xi, \varrho, \varphi)}{\left(\mho-\delta_{p}-\Delta \delta_{p}\right)\left(\mho-\delta_{p}\right)^{2}} d \mho$

But for all $\mho \in C_{1}$ and $\mathrm{z}$ is restricted to compact subset of $R^{n}, o<\hbar \leqslant \frac{\pi}{2}$

$A(z, \ell, f, w, p, u, \tilde{\delta}, \eta, \kappa, \xi, \varrho, \varphi)$

$=D_{z}^{E}\left\{K_{\lambda, \hbar}(z, \ell, f, w, p, u, \tilde{\delta}, \eta, \kappa, \xi, \varrho, \varphi)\right\}$

is bounded by a constant $\mathrm{G}$.

Moreover, $\left|\mho-\delta_{p}-\Delta \delta_{p}\right|>R_{1}-R>0$

and $\left|\mho-\delta_{p}\right|=R_{1}$. 
We have

$$
\begin{aligned}
& \left|D_{z}^{E} \mho_{\Delta_{p}}(z, \ell, f, w, p, u)\right| \\
& =\left|\frac{\delta_{p}}{2 \pi i} \int_{C_{1}} \frac{A(z, \ell, f, w, p, u, \tilde{\delta}, \eta, \kappa, \xi, \varrho, \varphi)}{\left(\mho-\delta_{p}-\Delta \delta_{p}\right)\left(\mho-\delta_{p}\right)^{2}} d \mho\right| \\
& \leq \frac{\left|\Delta \delta_{p}\right|}{2 \pi} \int_{C_{1}} \frac{G}{\left(R_{1}-R\right) R_{1}^{2}}|d \mho| \\
& \leq \frac{\left|\Delta \delta_{p}\right| G}{\left(R_{1}-R\right) R_{1}}
\end{aligned}
$$

similarly,

$$
\begin{aligned}
& \left|D_{\ell}^{A} \mho_{\Delta_{q}}(z, \ell, f, w, p, u)\right| \leq \frac{\left|\Delta \Psi_{q}\right| H}{\left(R_{1}-R\right) R_{1}}, \\
& \left|D_{f}^{L} \mho_{\Delta_{r}}(z, \ell, f, w, p, u)\right| \leq \frac{\left|\Delta \Xi_{r}\right| T}{\left(R_{1}-R\right) R_{1}} .
\end{aligned}
$$

Where,

$$
\begin{aligned}
& B(z, \ell, f, w, p, u, \delta, \tilde{\eta}, \kappa, \xi, \varrho, \varphi) \\
& =D_{\ell}^{A} \mho_{\Delta_{p}}(z, \ell, f, w, p, u) \text { is a }
\end{aligned}
$$

bounded by constant $\mathrm{H}$.

and

$$
\begin{aligned}
& B(z, \ell, f, w, p, u, \delta, \eta, \tilde{\kappa}, \xi, \varrho, \varphi) \\
& =D_{f}^{L} \mho_{\Delta_{r}}(z, \ell, f, w, p, u) \text { is a }
\end{aligned}
$$

bounded by constant $\mathrm{T}$.

Thus as $\left|\Delta \delta_{p}\right| \rightarrow 0 .\left|D_{z}^{E} \mho_{\Delta_{p}}(z, \ell, f, w, p, u)\right|$ tends to zero uniformly of the compact subset of $R^{n}$, therefore it follows that $\mho_{\Delta_{p}}(z, \ell, f, w, p, u)$ converge in $E\left(R^{n}\right)$ to zero.

$\therefore F_{\lambda, \hbar}(\delta, \eta, \kappa, \xi, \varrho, \varphi)$ is differentiable with respect to $\delta_{p}, \Psi_{q}, \Xi_{r}$.

But this is true for all $\mathrm{p}=1,2---$,

$q=1,2,---, r=1,2,--\cdot$.

Hence $F_{\lambda, \hbar}(\delta, \eta, \kappa, \xi, \varrho, \varphi)$ is analytic on $C^{n}$.

$$
D_{\delta, \eta, \kappa}^{E, A, L}\left\{F_{\lambda, \hbar}(\delta, \eta, \kappa, \xi, \varrho, \varphi)\right\}
$$

$=<h(z, \ell, f, w, p, u)$,

$D_{\delta, \eta, \kappa}^{E, A}\left\{K_{\lambda, \hbar}(z, \ell, f, w, p, u, \delta, \eta, \kappa, \xi, \varrho, \varphi)\right\}>$.

\section{Examples}

The following are some examples of 3DFRFMT for finding of some functions:

Example 3.1. Show that

$$
\begin{aligned}
& {[3 \operatorname{DFRFMT(1)}](\beta, \gamma, \delta, \vartheta, \rho, \mu)} \\
& =\frac{\sqrt{2 \pi(1-i \cot \lambda)}}{\sqrt{\cot \lambda}} e^{\frac{i \pi}{2}} \\
& e^{\frac{i}{2}\left[\frac{3+\cos 2 \lambda}{\sin 2 \lambda}\right]\left(\beta^{2}+\gamma^{2}+\delta^{2}\right)}(i \tan \hbar)^{3 / 2} \\
& e^{-i \pi \tan \hbar\left(\vartheta^{2}+\rho^{2}+\mu^{2}\right)} \text {. }
\end{aligned}
$$

Proof. We have

$3 D F R F M T\{h(x, y, z, t, q, p)\}$

$$
\begin{aligned}
& (\beta, \gamma, \delta, \vartheta, \rho, \mu) \\
= & \int_{-\infty}^{\infty} \int_{-\infty}^{\infty} \int_{-\infty}^{\infty} \int_{0}^{\infty} \int_{0}^{\infty} \int_{0}^{\infty} c_{1 \lambda}
\end{aligned}
$$$$
e^{\frac{i}{2 \sin \lambda}\left[\left(x^{2}+y^{2}+z^{2}+\beta^{2}+\gamma^{2}+\delta^{2}\right) \cos \Lambda\right]}
$$$$
e^{\frac{i}{2 \sin \lambda}[-2(x \beta+y \gamma+z \delta)]}
$$$$
\frac{2 \pi i \vartheta}{t \sin \hbar}-1 \frac{2 \pi i \rho}{q \sin \hbar}-1 \frac{2 \pi i \mu}{p \sin \hbar}-1
$$

$e^{\frac{\pi i}{\tan \hbar}\left[\vartheta^{2}+\rho^{2}+\mu^{2}+\log ^{2} t+\log ^{2} q+\log ^{2} p\right]}$

$$
h(x, y, z, t, q, p) d x d y d z d t d q d p
$$

putting,

$$
\begin{aligned}
& \log t=\varpi \Rightarrow t=e^{\varpi} \Rightarrow d t=e^{\varpi} d \varpi \\
& \log q=\vartheta \Rightarrow q=e^{\vartheta} \Rightarrow d q=e^{\vartheta} d \vartheta \\
& \log p=\psi \Rightarrow p=e^{\psi} \Rightarrow d p=e^{\psi} d \psi \\
& \therefore[3 D F R F M T(1)](\beta, \gamma, \delta, \vartheta, \rho, \mu) \\
& =C_{1 \lambda} e^{\frac{1}{2}\left(\beta^{2}+\gamma^{2}+\delta^{2}\right) \cot \lambda} \\
& \int_{-\infty}^{\infty} \int_{-\infty}^{\infty} \int_{-\infty}^{\infty} \int_{0}^{\infty} \int_{0}^{\infty} \int_{0}^{\infty}(1) e^{\frac{i}{2}\left(x^{2}+y^{2}+z^{2}\right) \cot \lambda} \\
& e^{-i(x \beta+y \gamma+z \delta) \operatorname{cosec} \lambda} \quad\left(e^{\varpi}\right) \frac{2 \pi i \vartheta}{\sin \hbar}-1 \\
& \left(e^{\nu}\right) \frac{2 \pi i \nu}{\sin \hbar}-1 \\
& \left(e^{\psi}\right) \frac{2 \pi i \psi}{\sin \hbar}-1
\end{aligned}
$$




$$
\begin{aligned}
& \begin{array}{l}
e^{\frac{\pi i}{\tan \hbar}\left[\vartheta^{2}+\rho^{2}+\mu^{2}+\varpi^{2}+\nu^{2}+\psi^{2}\right]} \\
=e^{\frac{i}{2}\left(\beta^{2}+\gamma^{2}+\delta^{2}\right) \cot \lambda}\left[\frac{e^{\frac{i \pi}{4}} \pi}{\frac{\cot \lambda}{2}}\right]
\end{array} \\
& e^{\frac{i \beta^{2} \operatorname{cosec}^{2} \lambda+i \gamma^{2} \operatorname{cosec}^{2} \lambda+i \delta^{2} \operatorname{cosec}^{2} \lambda}{2 \cot \lambda}} \\
& e^{\frac{\pi i}{\tan \hbar}\left[\vartheta^{2}+\rho^{2}+\mu^{2}\right]} \int_{-\infty}^{\infty} \int_{-\infty}^{\infty} \int_{-\infty}^{\infty}(e)^{i X(\vartheta \varpi+\rho \nu+\mu \Psi)} \\
& (e)^{i Y\left(\varpi^{2}+\nu^{2}+\Psi^{2}\right)} d \varpi d \nu d \Psi \\
& \text { where, } \quad X=\frac{2 \pi}{\sin \hbar}, Y=\frac{\pi}{\tan \hbar} \\
& =\frac{\sqrt{2 \pi(1-i \cot \lambda)}}{\cot \lambda} e^{\frac{i \pi}{2}} e^{\frac{i}{2} \beta^{2}\left[\frac{3+\cos 2 \lambda}{\sin 2 \lambda}\right]} \\
& e^{\frac{i}{2} \gamma^{2}\left[\frac{3+\cos 2 \Lambda}{\sin 2 \lambda}\right]} e^{\frac{i}{2} \delta^{2}\left[\frac{3+\cos 2 \lambda}{\sin 2 \lambda}\right]} \\
& e^{\frac{\pi i}{\tan \hbar}\left[\vartheta^{2}+\rho^{2}+\mu^{2}\right]} \int_{-\infty}^{\infty}(e)^{i Y \varpi^{2}+i X \vartheta \varpi} d \varpi \\
& \int_{-\infty}^{\infty}(e)^{i Y \nu^{2}+i X \rho \nu} d \nu \int_{-\infty}^{\infty}(e)^{i Y \Psi^{2}+i X \mu \Psi} d \Psi \\
& =\frac{\sqrt{2 \pi(1-i \cot \lambda)}}{\cot \lambda} e^{\frac{i \pi}{2}} \\
& e^{\frac{i}{2}\left[\frac{3+\cos 2 \lambda}{\sin 2 \lambda}\right]\left(\beta^{2}+\gamma^{2}+\delta^{2}\right)}(i \tan \hbar)^{3 / 2} \\
& e^{\frac{-i \pi}{\sin \lambda} \frac{\sin ^{2} \lambda}{\cos \Lambda}\left(\vartheta^{2}+\rho^{2}+\mu^{2}\right)} \\
& =\frac{\sqrt{2 \pi(1-i \cot \lambda)}}{\sqrt{\cot \lambda}} e^{\frac{i \pi}{2}} \\
& e^{\frac{i}{2}\left[\frac{3+\cos 2 \lambda}{\sin 2 \lambda}\right]\left(\beta^{2}+\gamma^{2}+\delta^{2}\right)}(i \tan \hbar)^{3 / 2} \\
& e^{-i \pi \tan \hbar\left(\vartheta^{2}+\rho^{2}+\mu^{2}\right)} .
\end{aligned}
$$

Example 3.2. Show that

$$
\begin{aligned}
& {[3 \operatorname{DFRFMT\delta }(x-a, y-b, z-c} \\
& t-\xi, q-\zeta, p-\Psi)](\beta, \gamma, \Omega, \vartheta, \rho, \mu) \\
& =C_{1 \lambda} e^{\frac{i}{2 \sin \lambda}\left[\left(a^{2}+b^{2}+c^{2}+\beta^{2}+\gamma^{2}+\Omega^{2}\right) \cos \lambda\right]}
\end{aligned}
$$

$$
\begin{aligned}
& e^{\frac{i}{2 \sin \lambda}[-2(a \beta+b \gamma+c \Omega)]} \\
& \sum^{\frac{2 \pi i \vartheta}{\sin \hbar}-1} \frac{2 \pi i \rho}{\zeta \sin \hbar}-1 \frac{2 \pi i \mu}{\sin \hbar}-1 \\
& e^{\frac{\pi i}{\tan \hbar}\left(\vartheta^{2}+\rho^{2}+\mu^{2}+\log ^{2} \xi+\log ^{2} \zeta+\log ^{2} \Psi\right)} .
\end{aligned}
$$

\section{Proof:}

$$
\begin{aligned}
& {[3 D F R F M T\{h(x, y, z, t q, p)\}](\beta, \gamma, \Omega, \vartheta, \rho, \mu)} \\
& =C_{1 \lambda} e^{\frac{i}{2}\left(\beta^{2}+\gamma^{2}+\Omega^{2}\right) \cot \lambda} \\
& \int_{-\infty}^{\infty} \int_{-\infty}^{\infty} \int_{-\infty}^{\infty} \int_{0}^{\infty} \int_{0}^{\infty} \int_{0}^{\infty} e^{\frac{i}{2}\left(a^{2}+b^{2}+c^{2}\right) \cot \lambda} \\
& e^{-i(a \beta+b \gamma+c \Omega) \operatorname{cosec} \lambda} \\
& \frac{2 \pi i \vartheta}{\xi \sin \hbar}^{-1} \frac{2 \pi i \rho}{\zeta \sin \hbar}-1 \frac{2 \pi i \mu}{\mu \frac{\sin \hbar}{\sin }-1} \\
& e^{\frac{\pi i}{\tan \hbar}\left(\vartheta^{2}+\rho^{2}+\mu^{2}+\log ^{2} \xi+\log ^{2} \zeta+\log ^{2} \Psi\right)} \\
& h(x, y, z, t, q, p) d x d y d z d t d q d p \\
& \therefore[3 D F R F M T \delta(x-a, y-b \text {, } \\
& z-c, t-\xi, q-\zeta, p-\Psi)](\beta, \gamma, \Omega, \vartheta, \rho, \mu) \\
& =C_{1 \lambda} e^{\frac{i}{2 \sin \lambda}\left[\left(a^{2}+b^{2}+c^{2}+\beta^{2}+\gamma^{2}+\Omega^{2}\right) \cos \lambda\right]} \\
& \begin{array}{l}
e^{\frac{i}{2 \sin \lambda}[-2(a \beta+b \gamma+c \Omega)]} \\
\xi^{\frac{2 \pi i \vartheta}{\sin \hbar}-1} \zeta^{\frac{2 \pi i \rho}{\sin \hbar}-1} \mu \frac{2 \pi i \mu}{\sin \hbar}-1
\end{array} \\
& e \frac{\pi i}{\tan \hbar}\left(\vartheta^{2}+\rho^{2}+\mu^{2}+\log ^{2} \xi+\log ^{2} \zeta+\log ^{2} \Psi\right) .
\end{aligned}
$$

\section{Applications}

We give applications of 3DFRFMT for solving some kind of third-order partial differential equations.

Application 4.1. We consider the following nonhomogeneous Mboctara partial differential equation [12]

$$
\partial_{z l f t m n} u(z, l, f, t, m, n)+u(z, l, f, t, m, n)=1 .
$$

Now applying 3DFRFMT in both side of the above equation, then we get 


$$
\begin{aligned}
& \frac{-2 \pi i}{\sin \hbar}\left[\left\{i \psi \operatorname{cosec} \Lambda \frac{\log t \cos \hbar}{t}\right\}\right. \\
& \text { FRFMT }[f(z, l, f, t . m . n)]-i\left(\Xi-\frac{\sin \hbar}{2 \pi i}\right) \cot \Lambda \\
& \operatorname{FRFMT}[f(z, l, f, t . m . n)]]+F R F M T[f(z, l, f, t . m . n)] \\
& =\frac{\sqrt{2 \pi(1-i \cot \Lambda)}}{\sqrt{\cot \Lambda}} e^{\frac{i \pi}{2}} e^{\frac{i}{2}\left[\frac{3+\cos 2 \Lambda}{\sin 2 \Lambda}\right]\left(\beta^{2}+\gamma^{2}+\delta^{2}\right)} \\
& (i \tan \hbar)^{3 / 2} e^{-i \pi \tan \hbar\left(\vartheta^{2}+\rho^{2}+\mu^{2}\right)} \\
& \text { or, } \frac{2 \pi}{\sin \hbar}\left\{i \psi \operatorname{cosec} \Lambda \frac{\log t \cos \hbar}{t}\right\} \\
& \text { FRFMT }[f(z, l, f, t . m . n)]-\frac{2 \pi}{\sin \hbar}\left(\Xi-\frac{\sin \hbar}{2 \pi i}\right) \cot \Lambda \\
& \text { FRFMT }[f(z, l, f, \text { t.m.n })] \\
& +F R F M T[f(z, l, f, t . m . n)] \\
& =\frac{\sqrt{2 \pi(1-i \cot \Lambda)}}{\sqrt{\cot \Lambda}} e^{\frac{i \pi}{2}} e^{\frac{i}{2}\left[\frac{3+\cos 2 \Lambda}{\sin 2 \Lambda}\right]\left(\beta^{2}+\gamma^{2}+\delta^{2}\right)} \\
& (i \tan \hbar)^{3 / 2} e^{-i \pi \tan \hbar\left(\vartheta^{2}+\rho^{2}+\mu^{2}\right)} \\
& \text { or, FRFMT[f(z,l,f,t.m.n)] } \\
& {\left[\frac{2 \pi}{\sin \hbar} i \psi \operatorname{cosec} \Lambda \frac{\log t \cos \hbar}{t}\right.} \\
& \left.-\frac{2 \pi}{\sin \hbar}\left(\Xi-\frac{\sin \hbar}{2 \pi i}\right) \cot \Lambda+1\right]=\frac{\sqrt{2 \pi(1-i \cot \Lambda)}}{\sqrt{\cot \Lambda}} e^{\frac{i \pi}{2}} \\
& e^{\frac{i}{2}\left[\frac{3+\cos 2 \Lambda}{\sin 2 \Lambda}\right]\left(\beta^{2}+\gamma^{2}+\delta^{2}\right)} \\
& (i \tan \hbar)^{3 / 2} \\
& e^{-i \pi \tan \hbar\left(\vartheta^{2}+\rho^{2}+\mu^{2}\right)} .
\end{aligned}
$$

Now applying the inverse three dimensional fractional FourierMellin transform, we obtain the following solution,

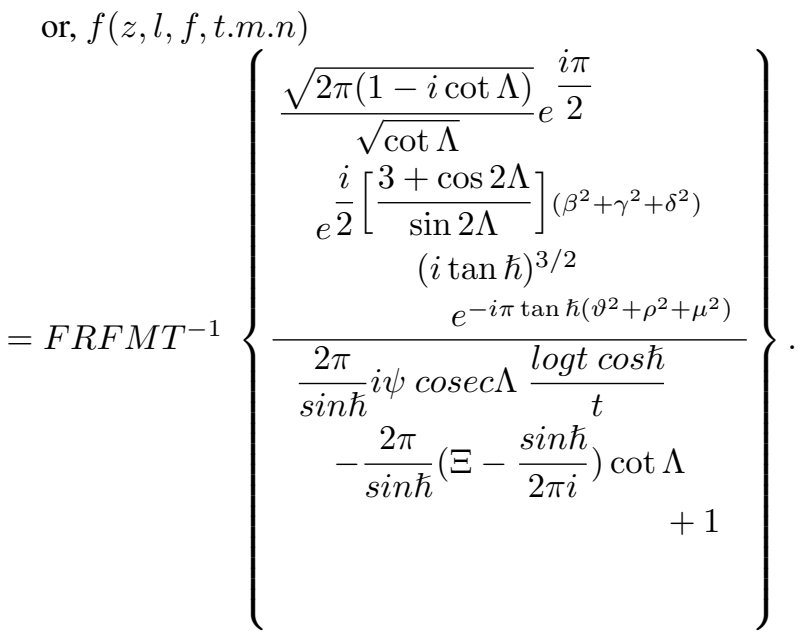

Application 4.2. We consider the following homogeneous
Mboctara partial differential equation [12]

$$
\partial_{z l f t m n} u(z, l, f, t, m, n)+u(z, l, f, t, m, n)=0 .
$$

Now applying three dimensional fractional Fourier-Mellin transform in both side of the above equation, then we get

$$
\begin{aligned}
& \frac{-2 \pi i}{\sin \hbar}\left[\left\{i \psi \operatorname{cosec} \Lambda \frac{\log t \cos \hbar}{t}\right\}\right. \\
& F R F M T[f(z, l, f, t . m . n)]-i\left(\Xi-\frac{\sin \hbar}{2 \pi i}\right) \cot \Lambda \\
& F R F M T[f(z, l, f, t . m . n)]] \\
& +F R F M T[f(z, l, f, t . m . n)]=0 \\
& \text { or, } \frac{2 \pi}{\sin \hbar}\left\{i \psi \operatorname{cosec} \Lambda \frac{\log t \cos \hbar}{t}\right\} \\
& F R F M T[f(z, l, f, t . m . n)] \\
& -\frac{2 \pi}{\sin \hbar}\left(\Xi-\frac{\sin \hbar}{2 \pi i}\right) \cot \Lambda \\
& F R F M T[f(z, l, f, t . m . n)] \\
& +F R F M T[f(z, l, f, t . m . n)]=0 \\
& \text { or, } F R F M T[f(z, l, f, t . m . n)] \\
& {\left[\frac{2 \pi}{\sin \hbar} i \psi \operatorname{cosec} \Lambda \frac{\log t \cos \hbar}{t}\right.} \\
& \left.-\frac{2 \pi}{\sin \hbar}\left(\Xi-\frac{\sin \hbar}{2 \pi i}\right) \cot \Lambda+1\right]=0 . \\
& \quad \text { Discussion and Conclusion } \\
& F R
\end{aligned}
$$

\section{Discussion and Conclusion}

Fractional Fourier transforms (FRFT) is a generalization of the Fourier transforms, which turned into resolving particular case of ODE's and PDE's as a signal processing kit. FRFT is called an effective tool for analyzing time-various indicators, especially in optical theory. FRFT has observed utility in signal processing, including filtering, fractional correlation, beam forming, non-compulsory filter out, convolution, filtering, and wavelet transforms time-frequency representation. In each vicinity in which Fourier transform and frequency domain concepts are used, the potential for generalization is progressed by employing the FRFT. The fractional Mellin transform (FRMT) is a generalization of the scale covariant remodel and the Mellin transform on the size warped timefrequency plane.

The fractional Fourier Mellin-based correlators may be used in detecting or controlling the range of moves. Additionally, each the conventional and the fractional-based correlators may be readily applied in optical theory, consequently finding correlated photos immediately at image acquisition time.

After obtaining the generalization, it can explore many more concepts in applying fractional Fourier-Mellin transfor- 
mation. Thus we have introduced a three-dimensional fractional Fourier-Mellin transforms (3DFRFMT) and investigated the modulation theorem, Parseval's theorem, analytic theorem, scaling theorem of three-dimensional fractional Fourier-Mellin transform. We have also given some examples of 3DFRFMT on some functions, which can also be applied to solve the different types of problems in any signal processing system, especially in a maritime strategy co-relator control moments specific three dimensional space. The work has been applied to solve the homogeneous and non-homogeneous Mboctara partial differential equations. The work may be useful for finding the surface design by solving third order homogeneous and non-homogeneous Mboctara partial differential equations by using the proposed operator.

\section{Acknowledgments}

The authors are extremely thankful to Department of Mathematics, National Institute of Technology Raipur (C.G.)-492010, India, for providing facilities, space and an opportunity for the work.

\section{Disclosure statements}

The authors declare that there is no conflict of interest.

\section{REFERENCES}

[1] L. Debnath and D. Bhatta. Integral Transform and their Applications, CRC Press, London, New-York, 2007.

[2] M.P. Goswami and N. Jha. Triple Laplace transform in biocomplex space with applications, Mathematics and Statistics, Vol. 8, No. 4, 443-450, 2020, DOI: 10.13189/ms.2020.080411
[3] M. Iii, J. Blazar and Z. Ayati. Mellin transform and conformable fractional operator: applications, Sema Journal, Vol. 5, 203$215,2019$.

[4] A. Zayed. Two dimensional fractional Fourier transform and its properties, Integral Transform and Special Functions, Vol. 29, 553-570, 2018.

[5] A. Kilicman and M. Omran. Note on fractional Mellin transform and applications, Springer Plus, Vol. 5, 2-8, 2016.

[6] V.L. Leontiev. Fourier method in initial boundary value problems for regions with curvilinear boundaries, Mathematics and Statistics, Vol. 9, No. 1, 24-30, 2021, DOI: 10.13189/ms.2021.090104.

[7] V.L. Leontiev. Orthogonal splines in approximation of functions, Mathematics and Statistics, Vol. 8, No. 2, 167-172, 2020, DOI: $10.13189 / \mathrm{ms} .2020 .080212$.

[8] V.D. Sharma and P.B. Deshmukh. Application of two dimensional fractional Mellin transform, International Journal of Scientific and Innovative Mathematical Research, Vol. 2, No. 9, 794-799, 2014.

[9] V.D. Sharma. Application of generalized two dimensional fractional Fourier transform, International Journal of Science and Research, Vol. 3, No. 9, 638-641, 2014.

[10] V.D. Sharma and P.B. Deshmukh. Modulation and parseval's identity of two dimensional fractional Fourier-Mellin transform, International Journal of Innovative Research in Science, Engineering and Technology, Vol. 5, 47-53, 2016.

[11] S.D. Manjarekar and A.P. Bhadane. Study of fractional transform and its applications, International Journal of Universal Mathematics and Mathematical Sciences, Vol. 2, No. 1, 53-61, 2016.

[12] A. Atanga. A note on the triple Laplace transform and its applications to some kind of third-order differential equations, Abstaract and Applied Analysis, Vol. 2013, Article ID 769102, 10 pages, 2013. 